\title{
The von Economo neurons in frontoinsular and anterior cingulate cortex in great apes and humans
}

\author{
John M. Allman • Nicole A. Tetreault • Atiya Y. Hakeem • Kebreten F. Manaye • \\ Katerina Semendeferi · Joseph M. Erwin · Soyoung Park • Virginie Goubert • Patrick R. Hof
}

Received: 1 December 2009/Accepted: 21 April 2010/Published online: 29 May 2010

(C) The Author(s) 2010. This article is published with open access at Springerlink.com

\begin{abstract}
The von Economo neurons (VENs) are large bipolar neurons located in frontoinsular (FI) and anterior cingulate cortex in great apes and humans, but not other primates. We performed stereological counts of the VENs in FI and LA (limbic anterior, a component of anterior cingulate cortex) in great apes and in humans. The VENs are more numerous in humans than in apes, although one gorilla approached the lower end of the human range. We also examined the ontological development of the VENs in FI and LA in humans. The VENs first appear in small numbers in the 36th week post-conception, are rare at birth, and increase in number during the first 8 months after birth. There are significantly more VENs in the right hemisphere than in the left in FI and LA in postnatal brains of apes and humans. This asymmetry in VEN numbers may be related to asymmetries in the autonomic nervous system. The
\end{abstract}

J. M. Allman $(\bowtie) \cdot$ N. A. Tetreault · A. Y. Hakeem ·

S. Park · V. Goubert

Division of Biology, 216-76, California Institute of Technology, Pasadena, CA 91125, USA

e-mail: cebus@caltech.edu

\section{K. F. Manaye}

Department of Physiology and Biophysics, College of Medicine, Howard University, Washington, DC 20059, USA

\section{K. Semendeferi}

Department of Anthropology, University of California,

San Diego, La Jolla, CA 92093, USA

\section{J. M. Erwin}

Biomedical Sciences and Pathobiology, Virginia Polytechnic Institute, Blackburg, VA 24036, USA

\section{P. R. Hof}

Department of Neuroscience, Mount Sinai School of Medicine, New York, NY 10029, USA activity of the inferior anterior insula, which contains FI, is related to physiological changes in the body, decisionmaking, error recognition, and awareness. The VENs appear to be projection neurons, although their targets are unknown. We made a preliminary study of the connections of FI cortex based on diffusion tensor imaging in the brain of a gorilla. The VEN-containing regions connect to the frontal pole as well as to other parts of frontal and insular cortex, the septum, and the amygdala. It is likely that the VENs in FI are projecting to some or all of these structures and relaying information related to autonomic control, decision-making, or awareness. The VENs selectively express the bombesin peptides neuromedin B (NMB) and gastrin releasing peptide (GRP) which are also expressed in another population of closely related neurons, the fork cells. NMB and GRP signal satiety. The genes for NMB and GRP are expressed selectively in small populations of neurons in the insular cortex in mice. These populations may be related to the VEN and fork cells and may be involved in the regulation of appetite. The loss of these cells may be related to the loss of satiety signaling in patients with frontotemporal dementia who have damage to FI. The VENs and fork cells may be morphological specializations of an ancient population of neurons involved in the control of appetite present in the insular cortex in all mammals. We found that the protein encoded by the gene DISC1 (disrupted in schizophrenia) is preferentially expressed by the VENs. DISC1 has undergone rapid evolutionary change in the line leading to humans, and since it suppresses dendritic branching it may be involved in the distinctive VEN morphology.

Keywords von Economo neurons - Fork cells . Anterior cingulate cortex - Frontoinsular cortex . Hominoid brain · Disc1 - Neuromedin B 


\section{Introduction}

In their comprehensive study of the cytoarchitecture of the human cerebral cortex, von Economo and Koskinas (1925) and von Economo (2009) described large bipolar neurons in frontoinsular (FI) cortex and in the limbic anterior (LA) area of anterior cingulate cortex. Von Economo (1926) called these specialized neurons the rod and corkscrew cells, referring to the straight and twisted variants of this distinct class of neurons. These unusual cells had previously been observed by many classical neuroanatomists including Betz (1881), Hammarberg (1895), and Ramón y Cajal (1899), but von Economo (1926) made a more complete description of their morphology and mapped their specific locations in human cortex. Rose (1927) additionally described cells of this type in anterior cingulate cortex of a chimpanzee brain. They have often been termed "spindle cells" (Nimchinsky et al. 1995; 1999), but because of possible confusion with other uses of this term, we have opted to call then von Economo neurons or VENs.

The VENs are projection neurons and are substantially larger than their pyramidal cell neighbors (Nimchinsky et al. 1999; Allman et al. 2001). They possess a single large basal dendrite, distinguishing them from pyramidal neurons, which have an array of smaller basal dendrites (Watson et al. 2006). This single large basal dendrite may have resulted from a transformation during evolution of the genetic programs for pyramidal neuron development to concentrate growth of the primary component of the basal dendrite and suppress the secondary and tertiary branching. The VENs have a narrow dendritic arborization that spans the layers of the cortex and may be able to sample and rapidly relay the output from a columnar array of neurons (Watson et al. 2006). The apical and basal dendrites of the VENs are remarkably symmetrical. This architecture suggests that these cells may be comparing inputs to these two symmetrical dendrites (Watson et al. 2006). The VENs in LA are filled retrogradely with the carbocyanin tracer DiI after it is deposited in the cingulum bundle, and thus the VENS are likely to be projection neurons with axonal connections extending through the underlying white matter to other parts of the brain (Nimchinsky et al. 1995). The large size of the VENs and their preferential staining with the antibody for non-phosphorylated neurofilament are also characteristic features of cortical projection neurons (Nimchinsky et al. 1995; Allman et al. 2002).

The posterior insula and anterior cingulate cortex in anthropoid primates receive differentiated inputs for pain, itch, warmth, cooling, and sensual touch, which are central components of highly evolved mechanisms for physiological homeostasis (Craig 2003, 2009). The VEN-containing areas FI, which is located in inferior anterior insula, and LA, which is a component of anterior cingulate cortex, may be considered as a further elaboration of these homeostatic mechanisms, which, while retaining some aspects of their basic physiological functions, such as appetite regulation, have extended to the regulation of social interactions and the homeostasis of interpersonal relationships. The inferior anterior insula has been found to be consistently activated by peripheral autonomic changes in an exhaustive metaanalysis of the imaging data (Mutschler et al. 2009). One such connection between autonomic arousal and decisionmaking is suggested by the findings of Critchley et al. (2000) who found that anterior insula was activated when the subjects had increased galvanic skin responses. The activity of anterior insula also increased as a function of uncertainty during the anticipatory period in a gambling task (Critchley et al. 2001a). Preuschhoff et al. (2008) found that the region of anterior insula closely matching the location of FI was specifically activated during "risk prediction error" when the consequences of a gambling decision in which a subject sustained a loss was revealed to the subject. The anterior inferior insula, in a location also closely corresponding to FI, is strongly activated by negative feedback in the form of frowning faces in a decision task involving a high degree of uncertainty (Ullsperger and von Cramon 2004). The region corresponding to FI on the right side is also activated when subjects scrutinize facial expressions to discern intentions (Baron-Cohen et al. 1999). The integrative functioning of the lateral part of FI in response to negative feedback is illustrated in a series of experiments by Jabbi et al. (2008) who elicited activity in this VEN-rich region through experiences involving disgust mediated by taste, by the observation of someone else responding to a disgusting taste, and by imagining a disgusting taste. (Note, however, that the activity observed in these experiments could also have arisen from the adjacent inferior frontal region.) These data together with many other experiments suggest that anterior insula and cingulate cortex are involved in the recognition of error and the initiation of adaptive responses to error and negative feedback (Gehring et al. 1993; Dehaene et al. 1994; Klein et al. 2007; Lamm and Singer 2010). Anterior insula and anterior cingulate cortex are major components of the system for the flexible control of goal-directed behavior (Dosenbach et al. 2007).

Anterior insula and anterior cingulate cortex are activated by situations that involve social error, a defect in the social network in which the individual is participating, or a change in state of one of the participants. For example, these structures are activated by resentment (Sanfey et al. 2003), deception (Spence et al. 2001), embarrassment (Berthoz et al. 2002), and guilt (Shin et al. 2000). They are also activated by feelings of empathy for the suffering of others, another type of social error signal (Singer et al. 2004a). In mothers, FI in the right hemisphere responds to 
the crying of distressed infants (Lorberbaum et al. 2002), which is a powerful social error signal. The anterior insula (including both superior and inferior components) was activated when partners in the prisoner's dilemma game failed to reciprocate co-operative moves made by the subject, which is a type of social error signal (Rilling et al. 2008). Anterior insula and anterior cingulate cortex are also activated by pro-social signals, such as love and trust (Bartels and Zeki 2004; Singer et al. 2004b), which suggests that these structures register both negative and positive aspects of the states of social networks. The responses of FI and LA are parametrically related to how humorous subjects judge cartoons to be; the humorous content of the cartoons typically involved social errors (Watson et al. 2007).

Recent work suggests that the anterior insula is involved in awareness (Ploran et al. 2007; Nelson et al. 2010; Craig 2010). This connection with awareness was initially suggested in an fMRI experiment by Kikyo and Ohki (2002) in which they observed activity in anterior insula when subjects reported the subjective sense of knowing a word before recalling it in a memory task, which these authors called the "feeling of knowing". More recently Ploran et al. (2007) found in an experiment employing a behavioral paradigm in which objects gradually emerge from noise that the activity of anterior insula was strongly linked to the moment when the subjects became aware of the identity of the object (allowing for the delays inherent in the fMRI signal). More recent work from the same group has identified several components of this activity, including one in inferior anterior insula particularly on the right side (Nelson et al. 2010). Allman et al. (2005) proposed that the VENs and related circuitry are involved in rapid intuition, which like perceptual recognition involves immediate effortless awareness rather than the engagement of deliberative processes. Recently, Aziz-Zadeh et al. (2009) found that when subjects solving anagrams arrived at rapid insightful solutions ("aha" moments) both anterior insula and anterior cingulate cortex were activated. These aspects of awareness are not limited to body states, but involve visual and linguistic experiences as well, suggesting the hypothesis that the role of anterior insula in awareness may include most or all aspects of perception and cognition. An extension of this hypothesis is that the VENs in FI serve as a fast relay of this information to other parts of the brain.

While these social processes are often conscious, they can also proceed covertly without the subject's direct awareness. For example, when subjects gaze into the eyes of others, if the size of the pupils of the eyes of the observed subject change in a way that is discordant with the changes in pupil size in the subject, both anterior cingulate and anterior insula are activated (Harrison et al. 2009). This discordance in pupil size change is reflective of differences in autonomic arousal and emotional experience between the observed individual and the subject. This is a covert signal that there is a discordance in their feelings about the social transaction that they are conducting, which is another type of social error signal. The use of Granger causality analysis of temporally resolved functional imaging data suggests that activity in FI precedes that in cingulate and other cortical areas, and Sridharan et al. (2008) proposed that the VENs may have a role in initiating this activity in other cortical areas. This initiation of activity may be related to the awareness of negative or positive feedback with respect to a broad range of internal and external states.

The VENs are implicated in several neuropsychiatric illnesses. Seeley et al. (2006, 2007; Seeley 2008) found in a stereological study that the VENs are specifically and selectively attacked in the early stages of the behavioral variant of fronto-temporal dementia (FTD), in which empathy, social awareness, and self-control are severely diminished. The VEN population of ACC is reduced by an average of $74 \%$ in these patients, and many of the surviving VENs are severely dysmorphic. The destruction of the VENs in FTD results, in different patients, from one of two distinct molecular mechanisms related to abnormal isoforms of the tau protein or to abnormal expression in the cytoplasm of VENs of the DNA-binding protein TDP-43 (Seeley et al. 2006; Seeley 2008). The VENs are also reduced in FI (Seeley et al. 2007). In contrast, Seeley et al. (2006, 2007) found that the VENs were not significantly reduced in Alzheimer's dementia (AD) although a reduction had been reported in an earlier study which did not use stereological methods (Nimchinsky et al. 1995). Rankin et al. (2006) systematically investigated the capacity for empathic concern in a large population of neurological patients and found that this capacity was greatly reduced in FTD patients; while this capacity was variably expressed in Alzheimer's patients it was not significantly reduced relative to neurologically normal controls.

Agenesis of the corpus callosum is another condition in which abnormal social behavior may be linked to reduced VEN populations. Patients with agenesis of the corpus callosum often have impoverished and superficial relationships, suffer from social isolation, and have interpersonal conflicts both at home and at work due to misinterpretation of social cues (Paul et al. 2007). Kaufman et al. (2008) found that the VENs were reduced by $50 \%$ in a subject with partial agenesis of the corpus callosum and $90 \%$ in a subject with complete agenesis, compared to adult controls. The VEN loss could not be attributed to the reduction or absence of the corpus callosum itself because the number of VENs in FI was found to be normal in another subject whose corpus callosum was destroyed as the result of a stroke 15 years before her death. Because the 
surgical sectioning of the corpus callosum does not disrupt social behavior (Shorvon 2005), the social deficit in agenesis of the corpus callosum may be related to VEN loss. We will present evidence that FI sends fibers into the corpus callosum, but apparently the severing of this connection does not result in a significant loss of VENs in FI.

There are many features of autism that suggest that the VENs may be involved in this disorder (Allman et al. 2005; van Kooten 2008). An initial stereological study of the numbers of VENs in area FI of four autistic subjects plus controls did not confirm this conjecture (Kennedy et al. 2007). However, a second stereological study of VENs in dorsal ACC involving nine autistic subjects plus controls found that the autistic subjects fell into two groups, one with significantly higher numbers of VENs than controls, and one with very few VENs (Simms et al. 2009). In this study the controls occupied a middle zone with respect to VEN counts with little overlap with the high or low VEN autistic groups. The results of Simms et al. (2009) suggest that two different mechanisms influence the number of VENs in autism, possibly through different effects on migration and survival. As in frontotemporal dementia, in autism the VENs may be vulnerable to more than one pathological process that may contribute to the disorder as it manifests in different individuals. A strong linkage between reduced activity in the right anterior insula in autistic subjects versus controls in social tasks contrasted with non-social tasks was revealed in a meta-analysis of 39 functional imaging studies (Di Martino et al. 2009). These studies suggest a possible role for the VENs in autism.

In a comparative study of the distribution of the VENs in LA in primates, Nimchinsky et al. (1999) found them to be present in all the great apes (chimpanzees, bonobos, gorillas, and orangutans), but not in any other non-human primates. In this study, the VENs declined in abundance with increasing phylogenetic distance from humans, with bonobos and chimpanzees having more than gorillas, which in turn had more than orangutans, which had only a few isolated VENs. However, this was based on qualitative observations, and no stereological determinations of VEN numbers or concentrations were performed. In the present study, we confirmed that among primates significant numbers of VENs are found in the FI region only in great apes and humans, and we performed stereological counts of the VENs both in FI and in LA in these species. (By significant numbers we mean more than 2 such cells per section through the FI region.) We also examined the ontological development of the VENs both in FI and in LA and found that they mainly emerge during the first 8 months after birth. In postnatal FI and LA, the VENS are nearly always more numerous in the right hemisphere than in the left both in apes and in humans. We found that a subset of the VENs and a related population of neurons, the fork cells, are strongly labeled by antibodies to the proteins neuromedin B (NMB) and gastrin releasing peptide (GRP) which are involved in appetite regulation, suggesting a phylogenetically conserved function of these cells. The VENs are also strongly labeled by an antibody to the protein encoded by the gene DISC1 (disrupted in schizophrenia). This gene has undergone rapid evolutionary change in the line leading to humans (Crespi et al. 2007) and may be related to the distinctive VEN morphology. We did a preliminary study in a gorilla brain of the connections of frontoinsular cortex based on diffusion tensor imaging. This revealed connections with the frontal pole as well as with other parts of frontal and insular cortex, the septum, and the amygdala.

\section{Methods}

\section{Materials and tissue processing}

Many of the brains used in the stereological study were specially prepared for this purpose at the California Institute of Technology. We obtained two bonobo, one chimpanzee, three gorilla, and four orangutan brains from apes which had died of natural causes in zoos. The ape brains were scanned in a 9.4 Tesla Bruker magnet at the California Institute of Technology Brain Imaging Center. The structural MRIs were performed using a high-resolution 3D FLASH sequence which lasted $16 \mathrm{~h}$. The voxels in the structural MRIs were $250 \mu \mathrm{m}$ isotropic. Diffusion tensor imaging of the brain of a 27-year-old male gorilla was done with a high angular-resolution (PGHE) scan weighted isotropically along 72 diffusion directions. The DTIs were done in a series of 6 scans, lasting 36 h, averaged together to achieve high signal-to-noise ratio. The DTI voxels were $1 \mathrm{~mm}$ isotropic. We used FSL to make probabilistic tractography maps of connections proceeding from seed voxels with locations based on co-registered histological sections through FI and in the adjacent superior anterior insula in the gorilla (http://www.fmrib.ox.ac.uk/fsl/fdt/index.html) (Croxson et al. 2005). We obtained human area FI tissue from the NICHD Brain and Tissue Bank for Developmental Disorders. These brains were cryoprotected with sucrose, frozen on a specially designed microtome stage, and cut into serial $100-\mu \mathrm{m}$ thick coronal sections which were mounted on gelatinized slides. The cut face of the tissue block was photographed with each new section and used to assist in the accurate mounting of the sections. In general every 5 th section was stained with a cresyl violet Nissl stain, although in some instances we prepared every 2nd section through area FI. The ape brains were sectioned whole (except in the case of one bonobo and one orangutan, for which only one hemisphere was available) and a 
series through the entire brain was Nissl stained and mounted. The human tissue was provided in the form of single-hemisphere slabs spanning the regions of interest; for each brain a series through all provided tissue was Nissl stained and mounted.

We also used material in three brain collections for stereological and comparative study. Brains in the Yakovlev and Welker Collections at the National Museum of Health and Medicine were celloidin embedded, cut into 30-35 $\mu \mathrm{m}$ thick sections, and Nissl stained with thionine. The third collection was Katerina Semendeferi's at the University of California at San Diego. These brains were paraffin embedded, sectioned at $20 \mu \mathrm{m}$, and Nissl stained using a modification of the Gallyas silver stain for neuronal perikarya (Gallyas 1971; Merker 1983).

The stereology was performed with the optical fractionator probe using StereoInvestigator (MBF Bioscience, Williston, VT). For each sample we made a stereological estimate of the number of VENs in layers 3 and 5 (layer 4 is absent in FI and ACC). The VENs also extend into layers 2 and 6 in regions adjacent to where they are abundant in layers 3 and 5 in FI. We determined the regions of interest on the basis of the presence of VENs; these regions corresponded to FI and limbic cortex subdivision A (LA) in the cytoarchitectonic maps of von Economo and Koskinas (1925). The most robust criterion used by von Economo for the cytoarchitectonic identification of FI and LA was the presence of these specialized neurons, which he found nowhere else in the entire cerebral cortex (von Economo 2009). Von Economo did not illustrate the localization of FI in frontal sections, however Rose (1928) did make such plots for 14 planes in his very thorough study of insular cortex, and his agranular insular areas (ai1 through ai8) in human frontal sections are similar to the location of FI that we have found in humans. Moreover, Rose (1928) also cited the presence of lancet- and spindle-shaped cells in layer 5 as important criteria for identifying these areas. The VENs are not uniformly distributed in these areas and tend to be concentrated in the regions of greatest cortical curvature, as was observed by von Economo (1926). In most cases we also made a stereological estimate of the total neuron population within the same region of interest established for the VEN counts in each sample. In addition to our stereological estimates of VEN and neuron numbers, we used the StereoInvestigator software to assist us in plotting the locations of every VEN within representative sections through FI in a 39 -year-old male chimpanzee, a 27-year-old male gorilla and a 1.6-year-old human, which we used as guides for making photomicrographs illustrating the cytoarchitecture of FI. We also made threedimensional reconstructions of FI and LA superimposed on MR images of the 39-year-old male chimpanzee and a young adult female human brain. In the chimpanzee, we first aligned the frontal plane of our MR image sets with the frontal plane of our histological sections using the program IMAGE-J. We then co-registered the histological sections containing FI and LA with the matching MR images, plotted the location of these areas, and made threedimensional reconstructions using the program AMIRA (Visage Imaging). We did not have both MR images and histological sections for any human brain, so we estimated the location of these areas in an MR image set for a young adult human female brain provided by Kirsten Tillisch and Emeran Mayer at UCLA. These MR images were acquired with a Siemens Trio Tim 3 Tesla machine. The location of FI and LA were then reconstructed with AMIRA.

Immunohistochemistry and quantification

Fixed human frontoinsular cortex (FI) was obtained from the NICHD Brain and Tissue Bank and the Mount Sinai School of Medicine Brain Bank. Samples from a 39 and a 51-year-old male were sectioned at $50 \mu \mathrm{m}$ on a vibratome. Both individuals died suddenly of myocardial infarctions. The immunohistochemical method was adapted from Sherwood et al. (2004). The primary antibody concentration was optimized using rabbit anti-DISC1 (human) (1:100 Zymed cat. No. 40-6800; Zymed Laboratories, Inc., San Francisco, CA), NMB (1:100, Protein Tech), or GRP (1:1,000, Protein Tech). Control sections were incubated with no primary antibody and with the corresponding goat $\mathrm{IgG}$ at the same concentration as the primary antibody. For the DISC1 study, three sections from each individual were Nissl-counterstained with cresyl violet. For each section, layer 5 of FI was traced and the neurons therein were counted using StereoInvestigator. Neurons were classified as to whether they were VENs or non-VENs and whether or not they were immuno-reactive, and the percentages of positive profiles were calculated. As the entire region of FI was not available for each individual, stereological counts through the whole structure were not possible; the three sections from each specimen were sampled individually and the results averaged.

\section{Results}

The locations of the VEN-containing areas FI and LA are illustrated in three-dimensional transparent reconstructions of the right hemispheres of the brains of a chimpanzee (Fig. 1) and a human (Fig. 2). These figures also show the location of FI and LA in frontal, horizontal, and parasagittal MRI sections that intersect on FI. In Fig. 3, every VEN in a section through FI and adjacent cortex from a chimpanzee, a gorilla, and a human is plotted. To the left of each section is a corresponding low-power photomicrograph of the section. 
Fig. 1 The location of the VEN-containing regions FI and LA in MR scans of the right hemisphere of a 39-year-old male chimpanzee. Locations were based on examination of Nissl-stained histological sections of this brain. LA and FI are labeled in red. $\mathbf{a}$ and $\mathbf{b}$ are lateral and medial views of a three-dimensional reconstruction of the right hemisphere. c Frontal, horizontal, and parasagittal MRI sections that intersect in FI. The frontal section corresponds to approximately the same level as the partial section from this individual illustrated in Fig. 3
A

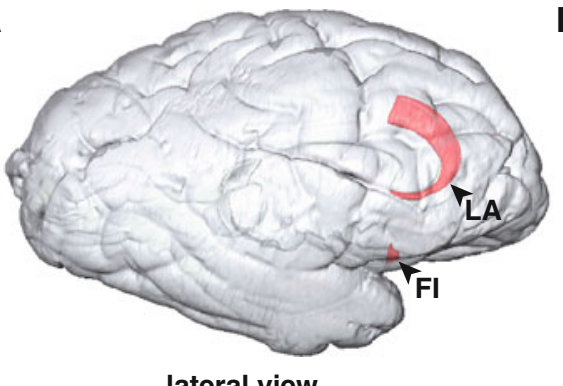

lateral view
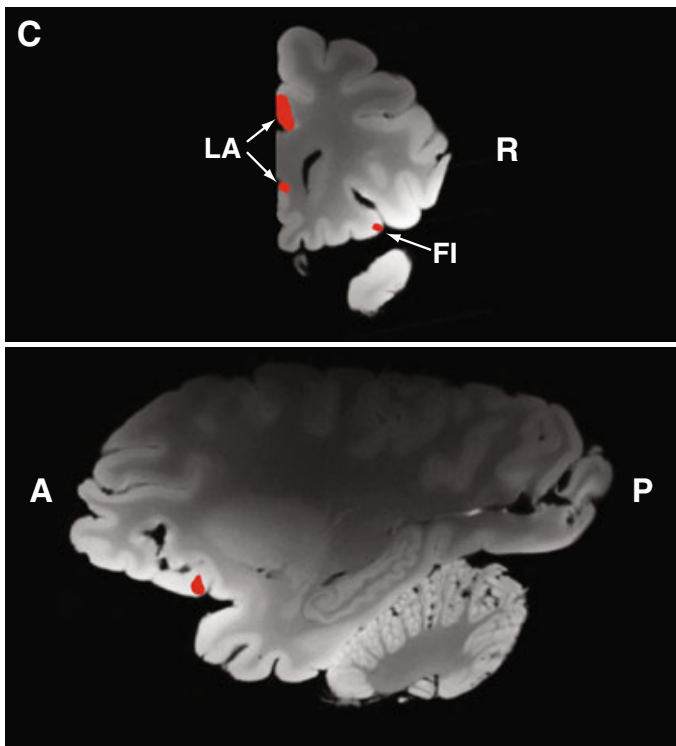

B

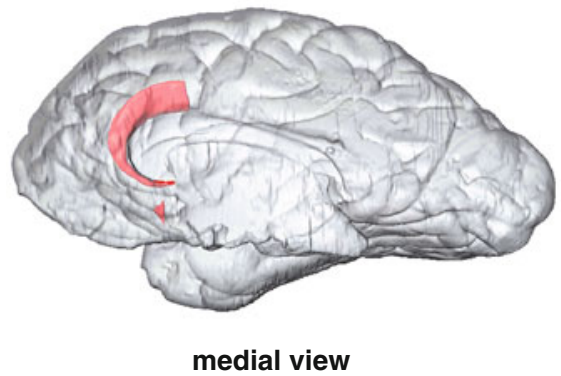

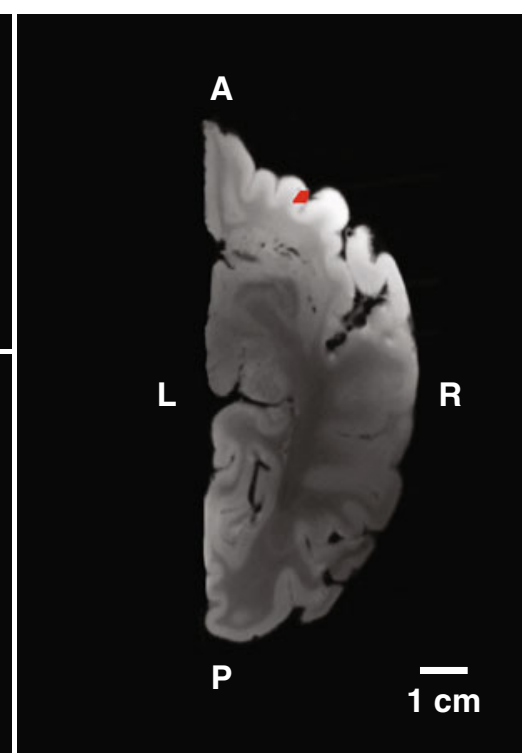

The section illustrated from the chimpanzee brain in Fig. 3 corresponds approximately to the frontal MRI section for this same individual illustrated in Fig. 1a. The VEN-containing area is largely confined to the region of high flexure in the chimpanzee, but extends medially in the gorilla and even further in the medial direction in the human. The transition between FI and superior anterior insula (SAI) corresponds to a gradient in VEN density rather than a sharp border. There are a small number of VENs in layer 6 in the adjacent superior anterior insula in all three species; these layer 6 neurons have the distinctive VEN morphology, but are oriented parallel to the white-gray matter interface and are orthogonal to the typical radial VEN orientation. These horizontally oriented cells may thus have a different functional role than the VENs in FI. In Fig. 4, medium-power photomicrographs from layer 5 of the same chimpanzee, gorilla, and human illustrate the strong radial (columnar) patterning of layer 5 in FI with the presence of many VENs, and the relative absence of these features in the anterior superior insula (SAI). The locations of the medium-power images are indicated by the arrowheads superimposed on the low-power photomicrographs in Fig. 3. SAI appears to correspond to Rose's (1928) granular insular areas (i7, i8, and i9). The cytoarchitecture of area LA and the location of the VENs in this area of anterior cingulate cortex are illustrated in von Economo (2009) and Nimchinsky et al. (1995, 1999).

The VENs are illustrated at higher magnification in Fig. 5 which shows their very similar morphology in the great apes and humans. In primates, the VENs are present in FI only in great apes and humans (see Table 1). This is the same taxonomic distribution as was found for the VENs in LA (Nimchinsky et al. 1999), which suggests that the VENs emerged as a specialized neuron type in the common ancestor of great apes and humans, a primitive ape living in the Miocene period (Kunimatsu et al. 2007). However, in orangutans we found only one out of seven individuals examined to have a substantial VEN population in FI and LA. The VEN distribution in this individual, a 25-year-old Sumatran female (Pongo abelii), closely resembled the pattern seen in the African apes. In two of the others (adult male Bornean orangutans, Pongo pygmaeus), the VENs were not present in the FI region, but were abnormally located in the gyrus rectus, which is in the medial orbitofrontal cortex, where VENs are usually not present. In the other four orangutans examined (an adult male Sumatran, 
Fig. 2 The location of the VEN-containing regions FI and LA in MR scans of the right hemisphere of a young adult human female. LA and FI are labeled in red. $\mathbf{a}$ and $\mathbf{b}$ are lateral and medial views of a three-dimensional reconstruction of the right hemisphere. c Frontal, horizontal, and parasagittal MRI sections that intersect in FI
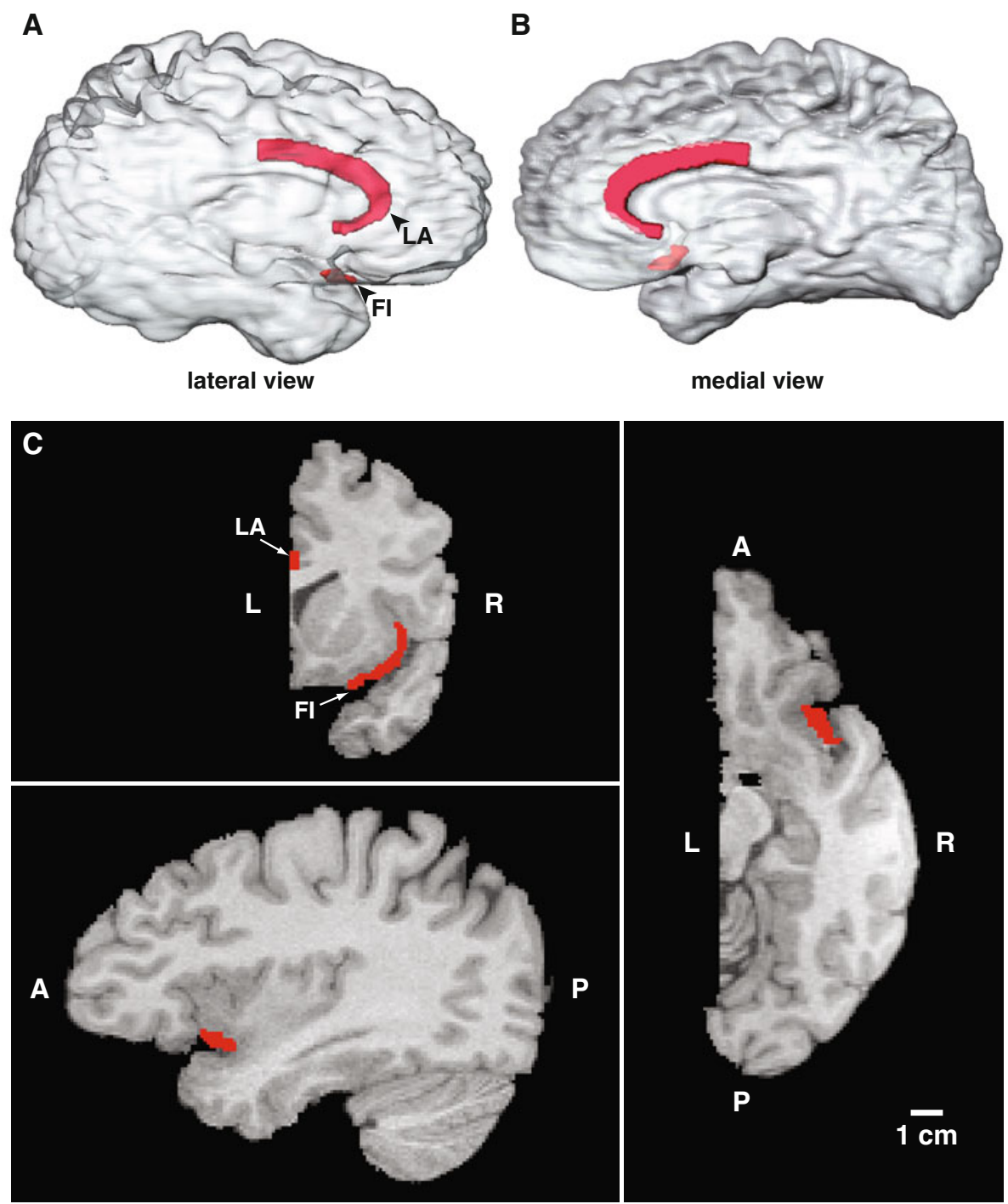

an adult male and a female Bornean, and an infant Bornean) VENs were rare both in FI and in LA.

The occurrence of VENs is not related to relative brain size or encephalization (Fig. 6). Some of the New World monkeys and lesser apes (gibbons) are much more encephalized than the great apes, but lack VENs. The presence of VENs may be related to absolute brain size, since the brain weights for the group on the left in Fig. 6 are much smaller than for the primates that have VENs (see Fig. 6 legend).

Tables 2 and 3 present the stereological data that we have collected for FI and LA in apes and humans. The key findings are also represented in the form of graphs (Figs. 7, 8,9 ). Figure 7 shows that the VENs are more numerous in humans than in apes, but that the VENs constitute a higher percentage of the total neurons in the regions of interest in the apes than in humans. In LA, one of the individual apes (a gorilla) stands out as having considerably more VENs than the others, approaching the lower end of the human range. The VEN count was similarly elevated relative to the other apes in this same gorilla in FI on the left side and also approached the lower end of the human range for this structure (see Table 2); unfortunately postmortem damage to the right FI in this individual made it impossible to make a stereological count for this structure in the right hemisphere. The relative abundance of VENs for this gorilla is also illustrated in comparison to a chimpanzee in Fig. 3. This individual gorilla had an exceptionally enriched environment (Patterson and Gordon 2002). Although we can conclude nothing definitive from this isolated observation, it does raise the possibility that VEN abundance may be related to environmental influences.

We examined FI and LA in fetal brains at postconception ages of 32 weeks $(n=1), 33$ weeks $(n=1)$, 34 weeks $(n=3)$, and 35 weeks $(n=2)$ and found no VENs. In one 36 week post-conception brain, small numbers of VENs were present in FI and LA. Figure 8 shows that the VENs exist in relatively low numbers in FI at birth; 

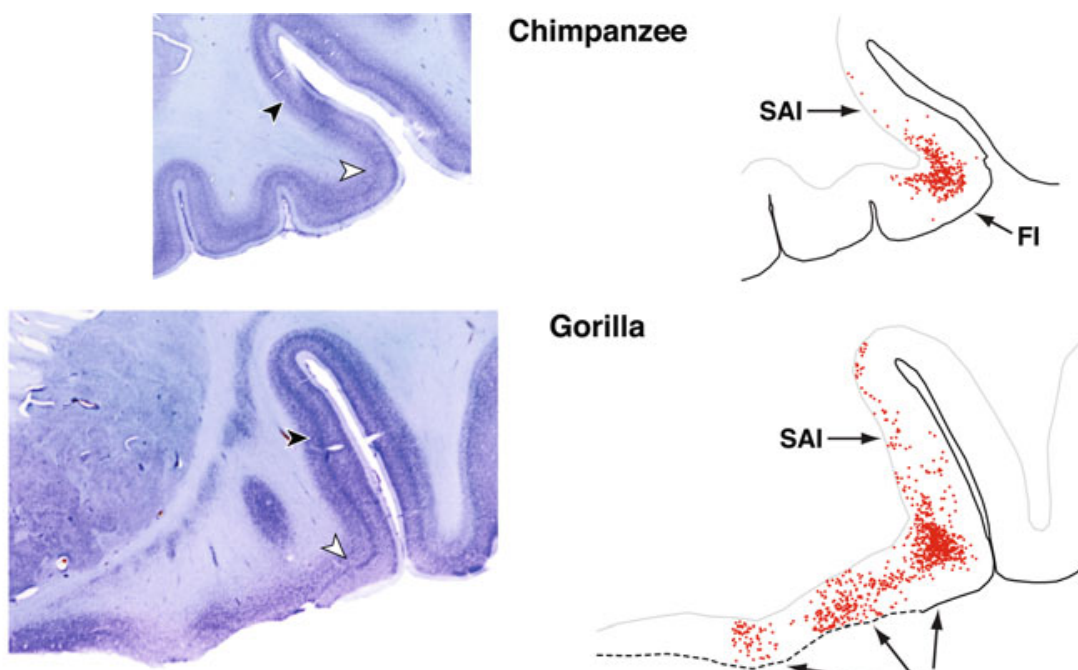

Gorilla
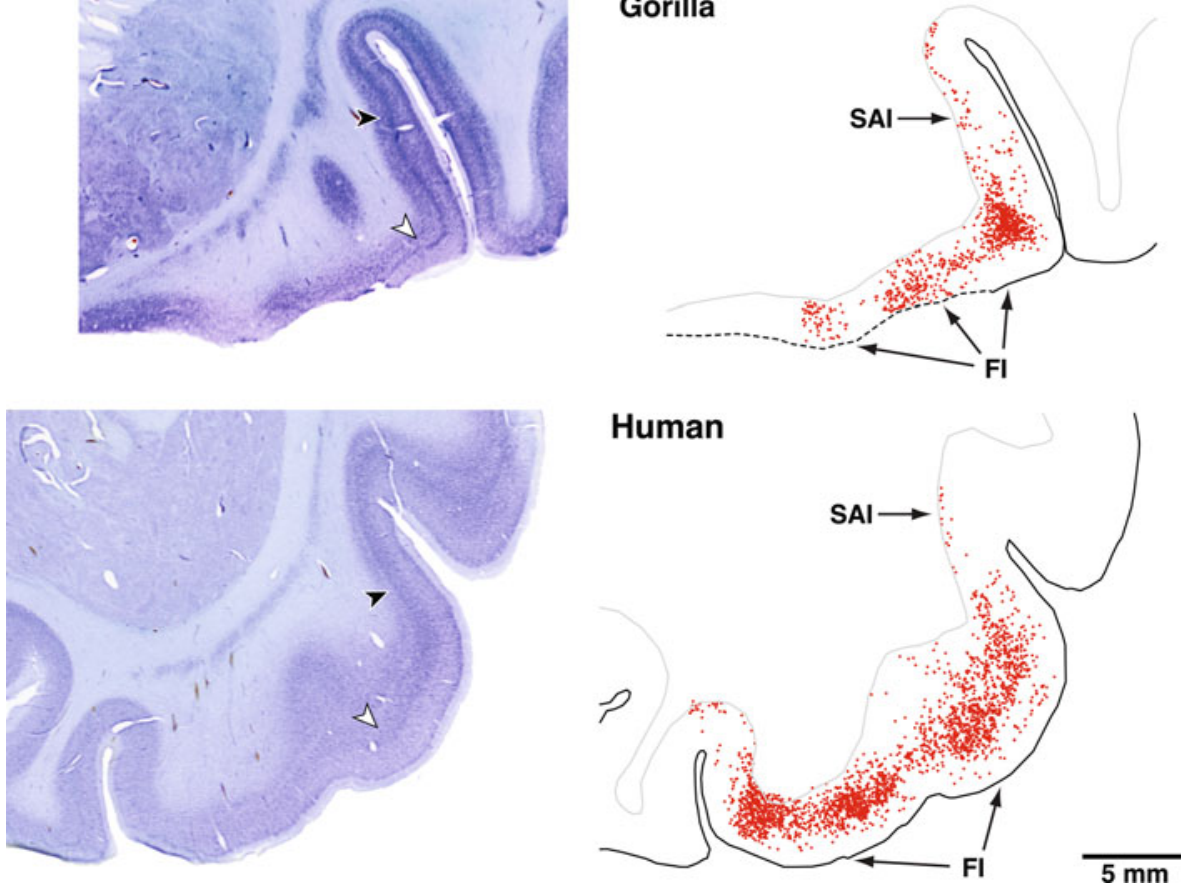

Fig. 3 Distribution of the VENs in area FI in a 39-year-old male chimpanzee, a 27-year-old male gorilla, and a 1.6-year-old male human. On the left side are photomicrographs of a section through the FI region in each individual. On the right side are outlines of these sections in which the location of each VEN has been plotted. There are 354 VENs plotted in the chimpanzee, 919 in the gorilla, and 2,415 in the human. Plots were made using a $40 \times$ oil immersion lens and total coverage was ensured by using the stereology program StereoInvestigator. Sections were $100 \mu \mathrm{m}$ thick. All images are

in LA the VENs were so rare that we were not able to make stereological estimates in the brains of neonates. In the late-term neonate (42 week post-conception) the number of VENs in FI was considerably higher than that in the normal-term neonates (38-40 week post-conception) suggesting that the number of VENs in FI increases immediately after the normal time of birth. The number of VENs is significantly greater in the postnatal brains relative to the neonatal brains. The percentage of total neurons that are VENs is relatively stable in adulthood (see Tables 2,3) and is similar to the percentages observed by Seeley et al. (2006).

Figure 9 illustrates the ratio between the number of VENs in the right hemisphere and that in the left. In newborns there is no clear hemispheric preference, but nearly all of the postnatal humans and all of the apes show

represented at the same scale. The sections are from right FI except for the gorilla, in which right FI was damaged during histological processing; left FI was substituted and the image reversed for ease of comparison with the other cases. The locations of the higher magnification photomicrographs shown in Fig. 4 are indicated on these sections by arrows (black for SAI, white for FI). The section of the gorilla corresponds approximately to Fig 12b, showing the location of the seed voxels for the tractography performed in this individual. FI frontoinsular cortex, SAI superior insular cortex

a clear predominance of VENs in the right hemisphere both in FI and in LA. The predominance is greatest in the 7 and 8-month-old infants. These data also suggest that the VENs develop in the right hemisphere before the left, which is consistent with the general pattern of the right hemisphere developing earlier than the left in embryogenesis (Gilles et al. 1983). In a large MRI study of adult humans, the cortex in the regions of FI and ACC was significantly larger on the right side than the left (Watkins et al. 2001).

Figure 10 shows immunocytochemical staining by an antibody to neuromedin B (NMB). The strong NMB staining was largely restricted to layer 5 (Fig. 10a). It is mainly the VENs which are NMB stained (Fig. 10b), although other classes of cells, including the fork cells and other neurons, are stained in layer 5 of FI in humans (Fig. 10c). We obtained similar staining in FI with 
Fig. 4 The cytoarchitecture of layer 5 of FI and SAI in chimpanzee, gorilla, and human. See Fig. 3 for the locations at which the photomicrographs were taken. Note the presence of the VENs and columnar architecture in the FI sections and the paucity of VENs and reduced columnar architecture in SAI. The sections are oriented so that the tops are closest to the pial surface. These images represent only one depth plane though the section, so only the VENs in focus at that depth plane are visible

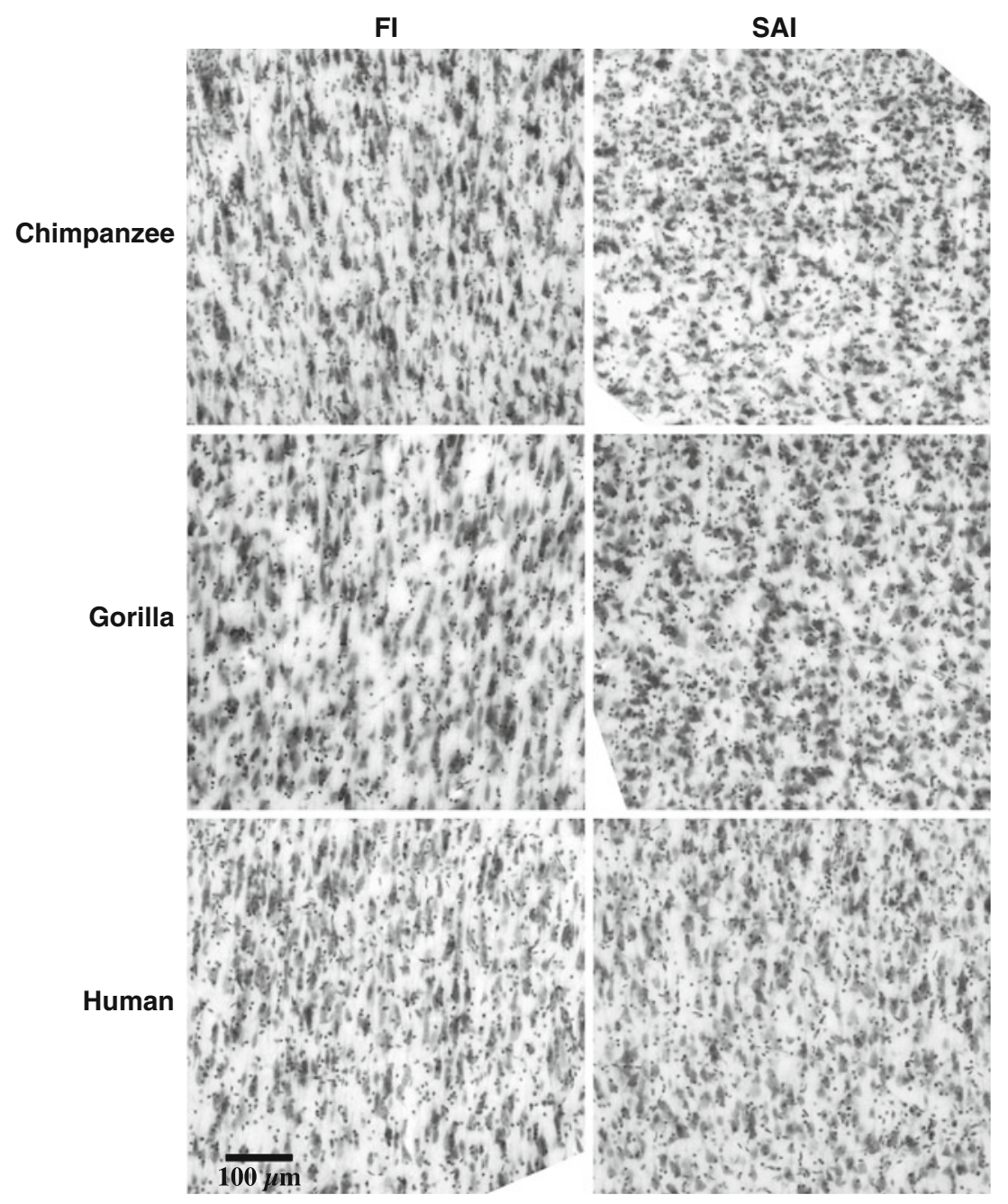

Fig. 5 VENs in area FI of humans and great apes. Photomicrographs are of Nisslstained sections. All panels share the scale indicated in the central panel

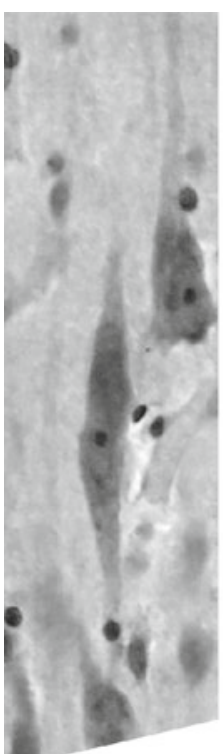

Human

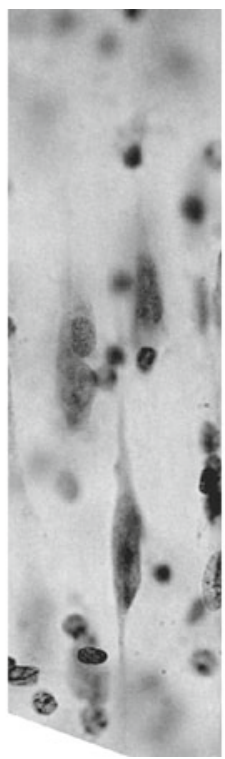

Bonobo

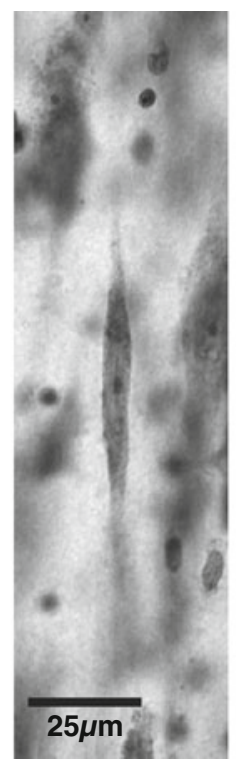

Chimpanzee

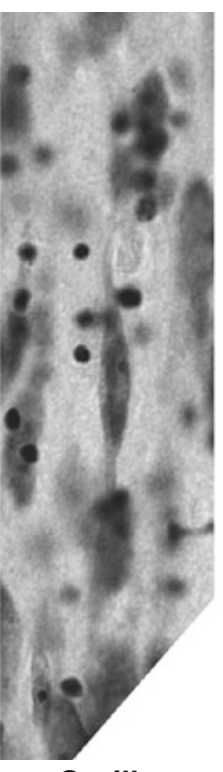

Gorilla

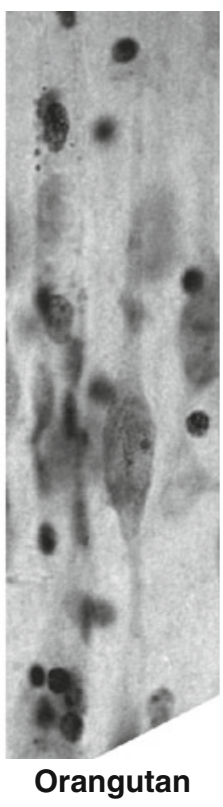


Table 1 Primate species examined for VENs in FI

\begin{tabular}{|c|c|c|c|}
\hline Taxonomy & Common name & VENs & $N$ \\
\hline \multicolumn{4}{|l|}{ Prosimii } \\
\hline \multicolumn{4}{|l|}{ Lemuroidea } \\
\hline \multicolumn{4}{|l|}{ Lemuridae } \\
\hline Hapalemur griseus & Lesser bamboo lemur & No & 1 \\
\hline Lemur catta & Ring-tailed lemur & No & 1 \\
\hline \multicolumn{4}{|l|}{ Indridae } \\
\hline Propithecus verreauxi & Verreaux’s sifaka & No & 1 \\
\hline \multicolumn{4}{|l|}{ Cheirogaleidae } \\
\hline Microcebus murinus & Gray mouse lemur & No & 1 \\
\hline \multicolumn{4}{|l|}{ Loroidea } \\
\hline \multicolumn{4}{|l|}{ Galagonidae } \\
\hline Galago senegalensis & Lesser galago & No & 1 \\
\hline \multicolumn{4}{|l|}{ Lorisidae } \\
\hline Loris tardigradus & Slender loris & No & 1 \\
\hline Nycticebus coucang & Slow loris & No & 1 \\
\hline Perodicticus potto & Potto & No & 1 \\
\hline \multicolumn{4}{|l|}{ Tarsioidea } \\
\hline \multicolumn{4}{|l|}{ Tarsiidae } \\
\hline Tarsius syrichta & Philippine tarsier & No & 1 \\
\hline \multicolumn{4}{|l|}{ Anthropoidea } \\
\hline \multicolumn{4}{|l|}{ Ceboidea } \\
\hline \multicolumn{4}{|l|}{ Callithricidae } \\
\hline Saguinus sp. & Tamarin & No & 1 \\
\hline \multicolumn{4}{|l|}{ Cebidae } \\
\hline Alouatta palliata & Mantled howler monkey & No & 1 \\
\hline Aotus trivirgatus & Owl monkey & No & 1 \\
\hline Ateles fusciceps & Black spider monkey & No & 1 \\
\hline Callicebus moloch & Dusky titi monkey & No & 1 \\
\hline \multicolumn{4}{|l|}{ Cercopithecoidea } \\
\hline \multicolumn{4}{|l|}{ Cercopithecidae } \\
\hline Cercocebus atys & Sooty mangabey & No & 1 \\
\hline Macaca mulatta & Rhesus macaque & No & 1 \\
\hline Mandrillus sphinx & Mandrill & No & 1 \\
\hline Papio papio & Guinea baboon & No & 1 \\
\hline Presbytis entellus & Hanuman langur & No & 1 \\
\hline \multicolumn{4}{|l|}{ Hominoidea } \\
\hline \multicolumn{4}{|l|}{ Hylobatidae } \\
\hline Hylobates lar & White-handed gibbon & No & 4 \\
\hline Hylobates syndactylus & Siamang & No & 1 \\
\hline \multicolumn{4}{|l|}{ Hominidae } \\
\hline Pongo pygmaeus & Bornean orangutan & Yes $^{\mathrm{a}}$ & 4 \\
\hline Pongo abelii & Sumatran orangutan & Yes $^{\mathrm{a}}$ & 3 \\
\hline Gorilla gorilla gorilla & Western lowland gorilla & Yes & 3 \\
\hline Pan troglodytes & Chimpanzee & Yes & 4 \\
\hline Pan paniscus & Bonobo & Yes & 4 \\
\hline Homo sapiens & Human & Yes & $16+$ \\
\hline
\end{tabular}

${ }^{\text {a }}$ See text

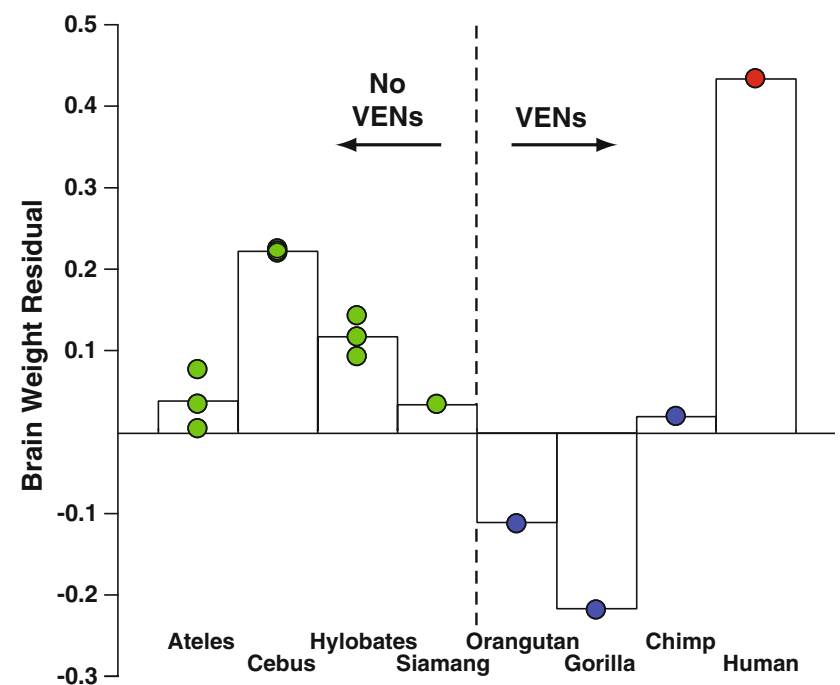

Fig. 6 Encephalization is not correlated with the presence or absence of VENs. Brain weight residuals were calculated by fitting a leastsquares regression line to a plot of brain weight versus body weight for a large sample of haplorhine primate species. The residual for a given species is the distance that species' brain weight falls above or below the regression line. Thus, a highly encephalized species, having an unusually large brain for its body size, will have a positive brain weight residual, while one with an unusually small brain for its body size will have a negative residual. The species to the left of the dashed line do not have VENs (see Table 1), but are more highly encephalized than the great apes (to the right of the line) which do have VENs. The bars indicate the average value of the data points in each column. The average brain weights are: Ateles $108.8 \mathrm{~g}$, Cebus $67.6 \mathrm{~g}$, Hylobates $94.4 \mathrm{~g}$, siamang $131.3 \mathrm{~g}$, orangutan $344.4 \mathrm{~g}$, gorilla $493.8 \mathrm{~g}$, chimpanzee $402.3 \mathrm{~g}$, and human $1,295 \mathrm{~g}$. Note that all the individuals to the left of the graph have brain weights below $150 \mathrm{~g}$, suggesting that possession of VENs may be related to an absolute brain size threshold. Brain and body weight data are from Stephan et al. (1981), and Brauer and Schober (1970). Also see Allman et al. (1993)

antibodies to another bombesin peptide, gastrin releasing peptide (GRP).

Figure 11 illustrates the strong immunocytochemical staining of the VEN somas and dendrites in area FI by an antibody to DISC1. This section was counterstained with cresyl violet so that cell bodies unlabeled by the DISC1 antibody appear blue. In the 51-year-old male we found that $90 \%$ of the VENs were DISC1-positive, while only $37 \%$ of the other layer 5 neurons were positive; in the 39-year-old male, 94\% of the VENs were DISC1-positive, whereas only $34 \%$ of the other neurons were positive. Thus, DISC1 is preferentially expressed in the VENs versus other layer 5 neurons in area FI.

Figure 12 illustrates tractography arising from seeds placed in FI and superior anterior insula of a gorilla brain in locations corresponding to sites illustrated for this 


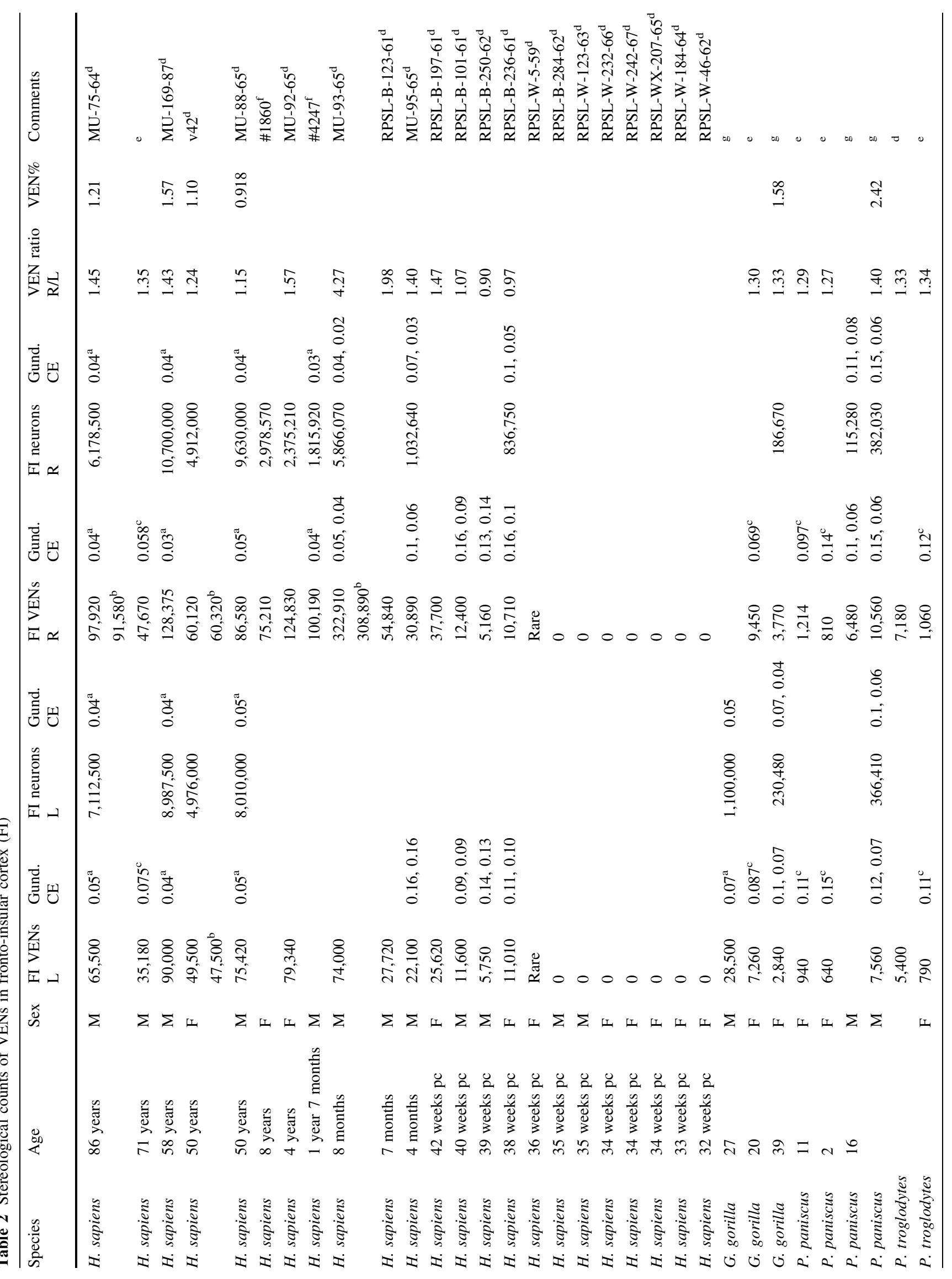




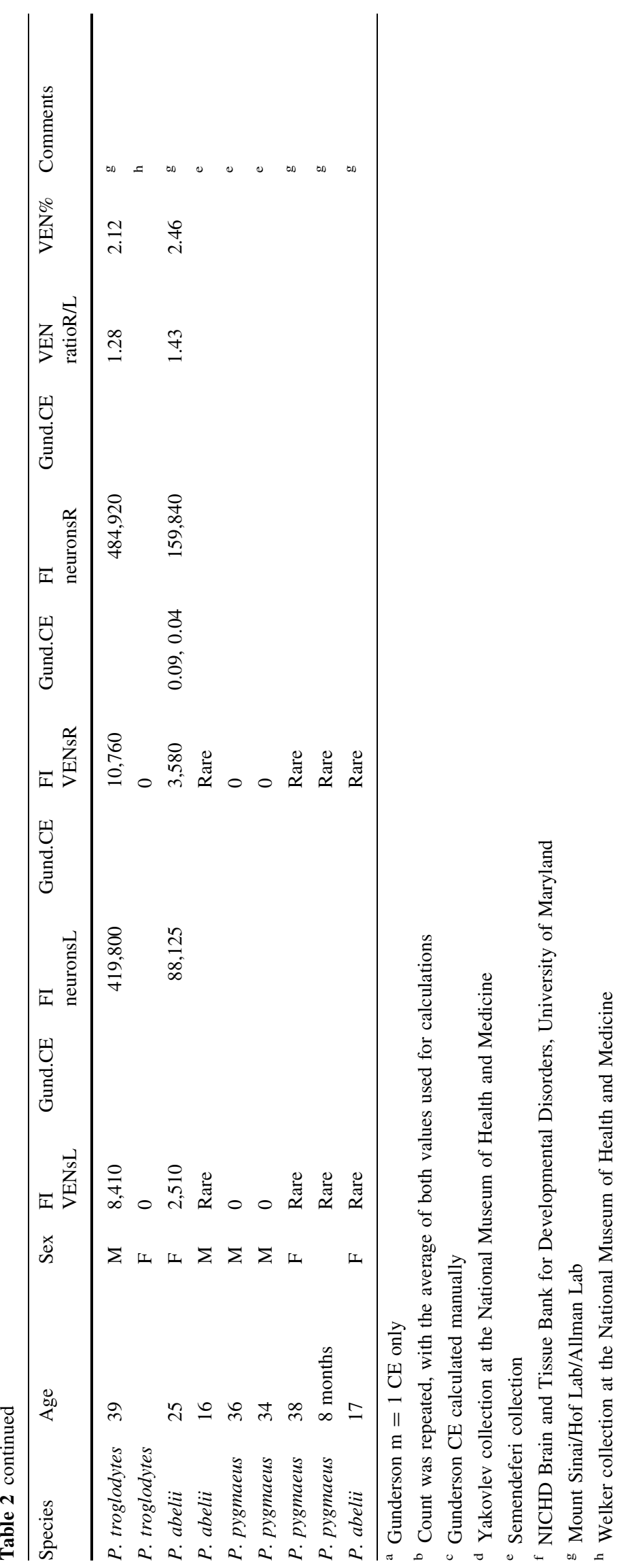




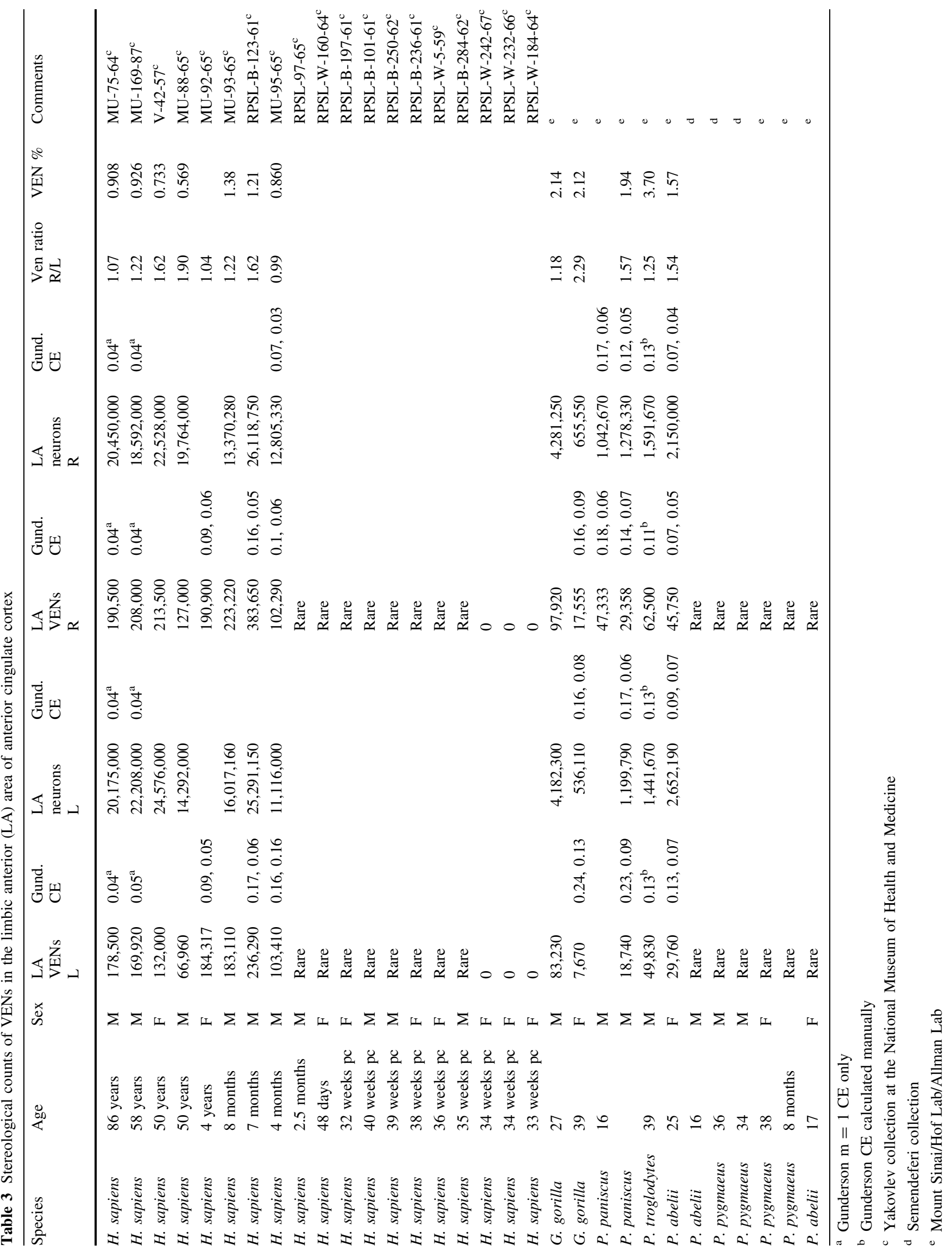


Fig. 7 A comparison of the number and proportion of VENs in areas FI and LA of adult humans and great apes. (See Tables 2, 3 for data.) Bars indicate the average of all data points in a given column. a The number of VENs in area FI (both hemispheres combined). FI contains significantly more VENs in humans than in great apes $(P=0.001)$. b The percentage of neurons in area FI that are VENs. Although the great apes have a smaller total number of VENs in FI, they have a significantly higher proportion of VENs to total neurons in FI $(P=0.029)$. c The number of VENs in LA (both hemispheres combined). As in area FI, humans have more VENs than do the great apes $(P=0.016)$, although this difference is smaller in LA. d The percentage of neurons in LA that are VENs. Again, as in FI, the great apes have a higher percentage of neurons that are VENs $(P=0.016)$. All comparisons are Mann-Whitney tests
A
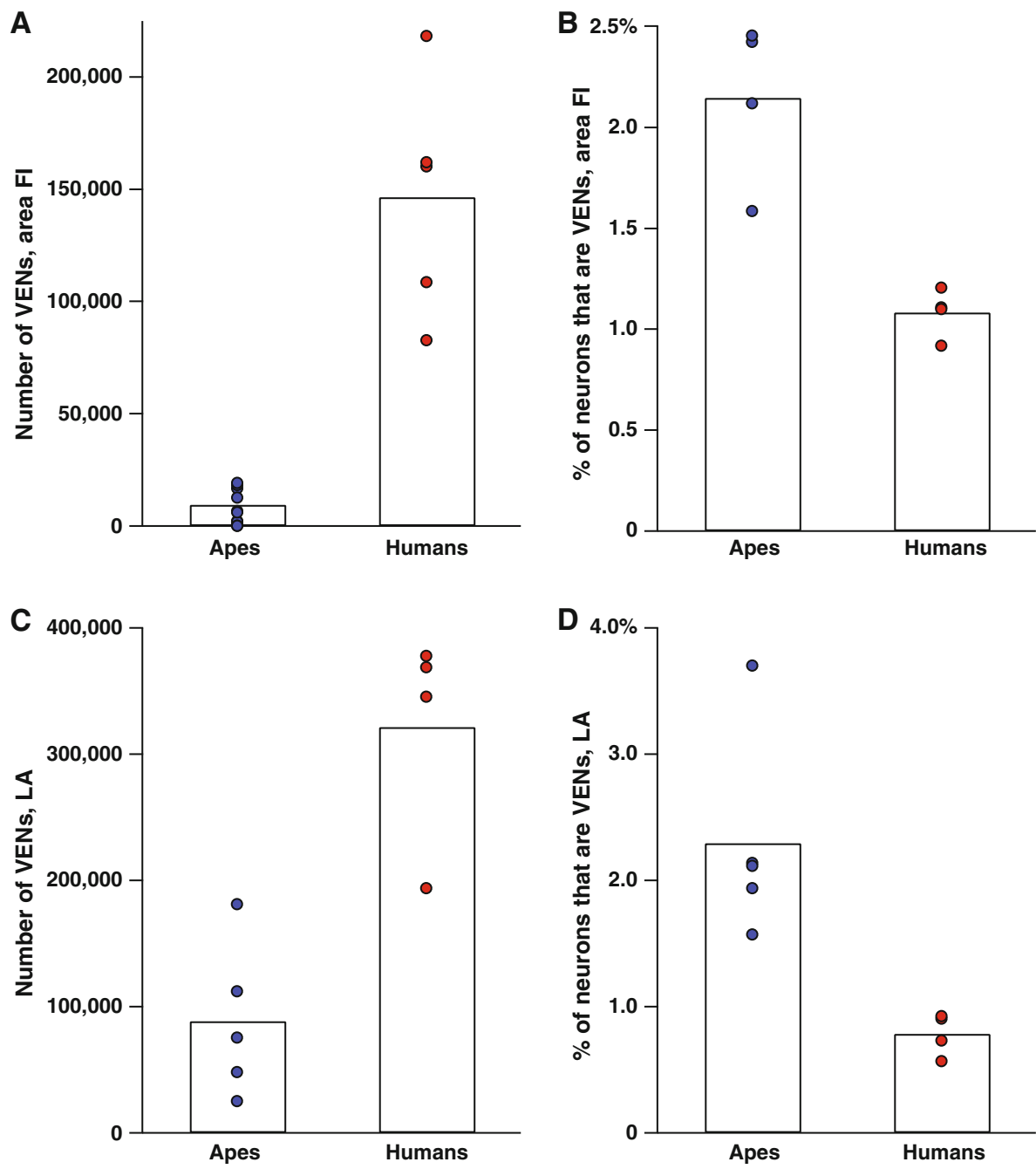

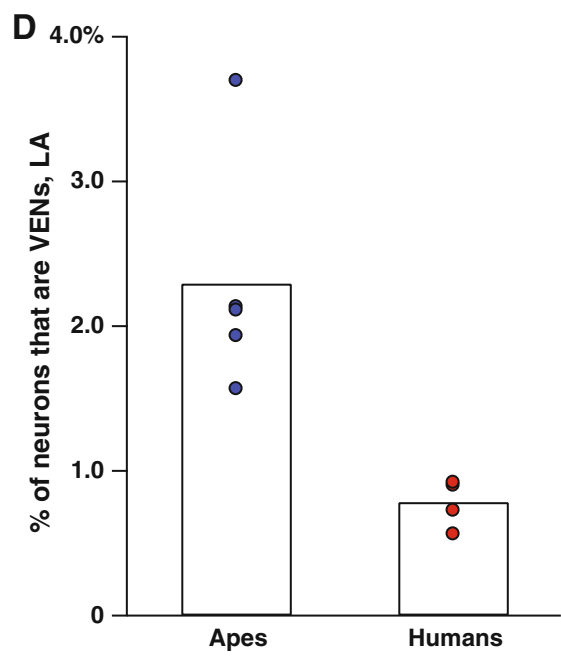

individual in Fig. 3. Figure 12a is a lateral view of the brain showing the levels depicted in $12 \mathrm{~b}$ through 1 . Each seed depicted in $12 \mathrm{~b}$ corresponds to a single voxel. The connections for the medial seed in FI are illustrated in green; the connections for the lateral FI seed are in red; the connections for the superior anterior insula seed are in blue. Where these connections overlap the connections are depicted as mixtures of these colors. Figure 12c through 1 shows the MR sections proceeding from anterior to posterior containing the color-coded tracts. Figure $12 \mathrm{c}$ and d show connectivity between the FI seeds and the frontal polar cortex present in both hemispheres, but stronger on the ipsilateral side. Figure 12e shows that the medial FI seed connection (green) has shifted laterally at this level and the lateral FI and superior AI seeds have more dorsal connections depicted in red and purple (for the overlap between red and blue) at this level. Figure $12 \mathrm{~g}$ and $\mathrm{h}$ show that the lateral FI and superior AI seeds are connected to the inferior frontal gyrus at these levels. Medial FI is connected with the septum, and lateral FI has connections in the corpus callosum at this level. Figure $12 \mathrm{j}$ shows connections between medial FI and the amygdala. Figure 12k shows lateral FI and superior AI connections with the posterior insula and medial FI with connections near inferotemporal cortex. Figure 121 shows lateral FI and superior FI connections just lateral to the hippocampus.

\section{Discussion}

The VENs are a phylogenetically recent specialization in hominoid evolution. The VENs in FI and in LA tend to be more numerous on the crowns of gyri, suggesting that the VEN-containing areas have undergone differential expansion reminiscent of other cortical specializations, such as the representations of the highly sensitive pads of the forepaw in the raccoon somatosensory cortex (Welker and Seidenstein 1959; Allman 2000). Especially in the human 


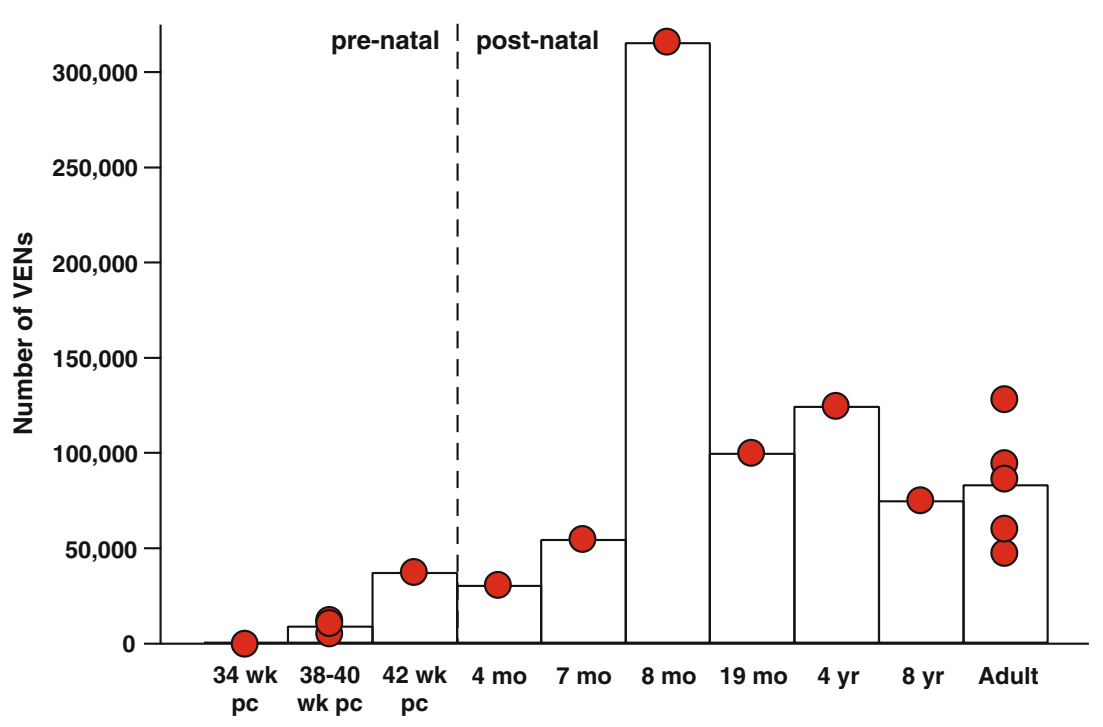

Fig. 8 The number of VENs increases after birth. The number of VENs in right FI in humans of different ages. VEN numbers are low in neonates and increase after birth. The 8-month-old individual examined had markedly more VENs in the right hemisphere than any other subject in this study; this might possibly be due to individual variation. The right hemisphere VEN measurement in this individual was repeated with similar results (see Table 2). The difference between the number of VENs in right FI for pre- and post-natal subjects was statistically significant $(P=0.0029)$, and this significance remained when the 8-month-old individual was removed from the comparison $(P=0.0040)$. The number of VENS in left FI and in both hemispheres together was also significantly different for pre- and post-natal individuals $(P=0.0056$ for both). Significance was determined using the Mann-Whitney test brain, there is considerable variability in the presence and number of small sulci and gyri within FI, and these influence the VEN distributions. The concentration of VENs in the crowns of small gyri is also a notable feature of FI in the elephant (Hakeem et al. 2009).

The possession of VENs in primates is not related to relative brain size or encephalization (see Fig. 6). Instead it appears to be related to absolute brain size. The VENs are present in primates with adult brain sizes greater than about $300 \mathrm{~g}$. They are also present in the apparent homologs of FI and LA in other mammals with very large brains, such as cetaceans and elephants (Hof and Van der Gucht 2007; Butti et al. 2009; Hakeem et al. 2009). Nearly all of these mammals are also highly social. We think that both large brain size and complex social behavior favor specialized neural systems for rapid communication within brain circuits. Large brains may be inherently slower because of the greater distances over which messages must be sent. Large brains also suffer from the limitations associated with packing large myelinated axons into a restricted space. However, fiber pathways in large brains have small sets of very large axons, which may serve as a compromise between the needs for rapid communication and the packing constraint (Wang et al. 2008). Thus, the evolution of the VENs may be an adaptation related to large brain size. Complex social behavior is often fast-paced, and this puts a premium on the capacity to respond quickly to changing conditions. A basic function of FI may be to register feedback crucial for initiating fast adaptive responses to changes. This would be consistent with the activity of FI preceding linked activity in ACC and other cortical areas (Sridharan et al. 2008).

We found two interesting differences between the distribution of VENs in humans and apes. The first difference between humans and apes is the relationship between area FI and agranular insular cortex, i.e. insular cortex lacking a layer 4. In humans, area FI, which is defined by the presence of VENs, appears to correspond to most of agranular insular cortex as delineated by Rose (1928). However, in apes area FI appears to correspond to a smaller part of the total agranular insular cortex. This difference may explain why there are typically considerably more VENs in humans than in apes. The second difference between humans and apes is that the density of VENs relative to other neurons in FI and LA is significantly higher in apes than in humans. One possible explanation for this surprising finding is that there may be other specialized neuronal populations that are differentially expanded in humans relative to apes. The presence and distribution of VENs are variable in orangutans, as are reports of their social behavior (Galdikas 1985; Mitani et al. 1991; Singleton and Van Schaik 2002), which range from solitary to relatively social, although typically orangutans are found to participate in smaller social groups than the other great apes. It is of interest that other parts of the neural systems underlying social behavior, such as the amygdala and the orbitofrontal cortex, are smaller or simpler in orangutans than in other great apes (Barger et al. 2007; Schenker et al. 2005; Semendeferi et al. 1998). 

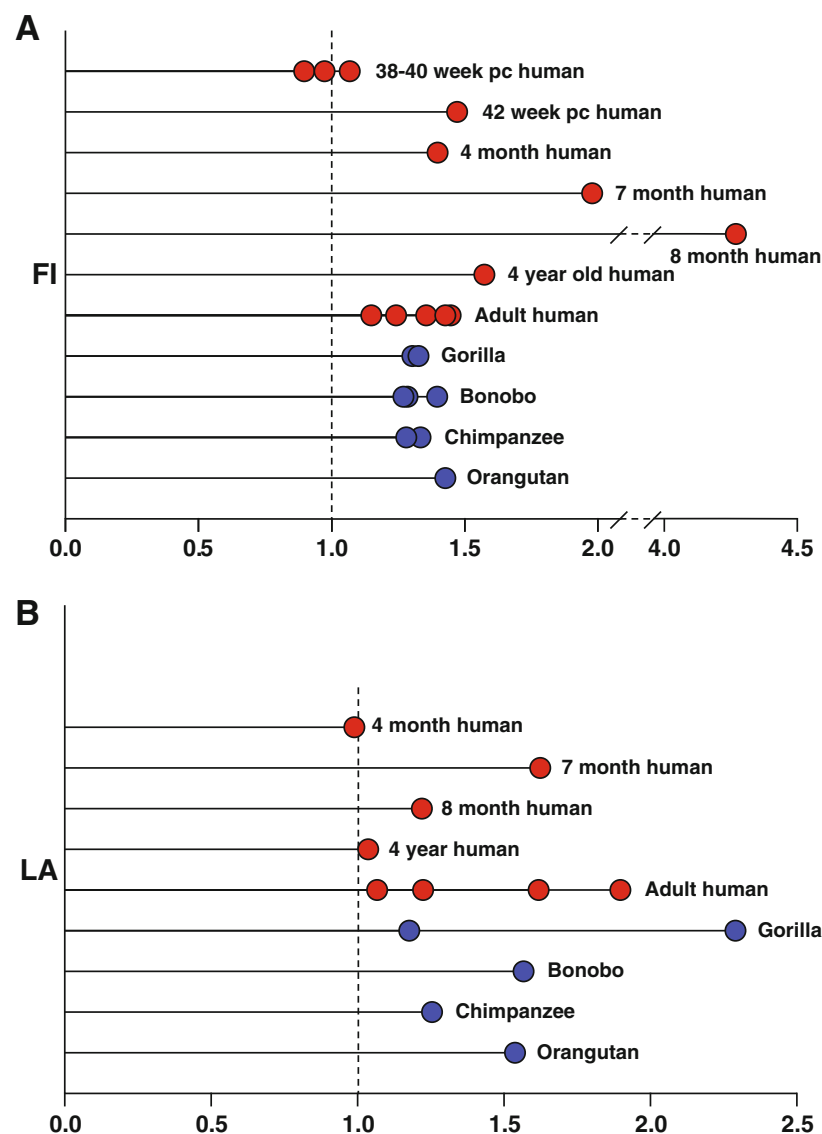

Fig. 9 The ratio of the number of VENs in the right hemisphere to the number of VENs in the left hemisphere. a In post-natal humans and great apes there are consistently more VENs in FI on the right side. This ratio develops after birth. In neonates, the numbers in each hemisphere are almost equal, while in infants, juveniles, and adults there are many more VENs in the right hemisphere. When the numbers of VENs in the right and left hemispheres of post-natal subjects were compared the difference was statistically significant both with and without the 8 -month-old outlier $(P=0.0039$ for all post-natal humans and $P=0.0078$ without the 8 -month-old subject). For post-natal apes and humans combined the hemispheric difference for FI was significant at $P<0.0001$. b The ratio of VENs in right and left LA. This ratio is less consistent than in area FI, but in almost all cases there are more VENs on the right side. When the number of VENs in the right and left hemispheres in post-natal humans was compared for LA, the result was statistically significant $(P=0.03)$. If post-natal apes and humans were combined, the difference was significant at $P=0.001$. Significance was determined using the Mann-Whitney test

\section{Postnatal emergence of the VENs}

The VENs mostly emerge postnatally, which can be seen in their numbers, concentrations, and the formation of the hemispheric predominance of VENs on the right side in the first few months after birth. This emergence could come about by the transformation of another cell type into the VENs or by postnatal neurogenesis. The long, thin spindle
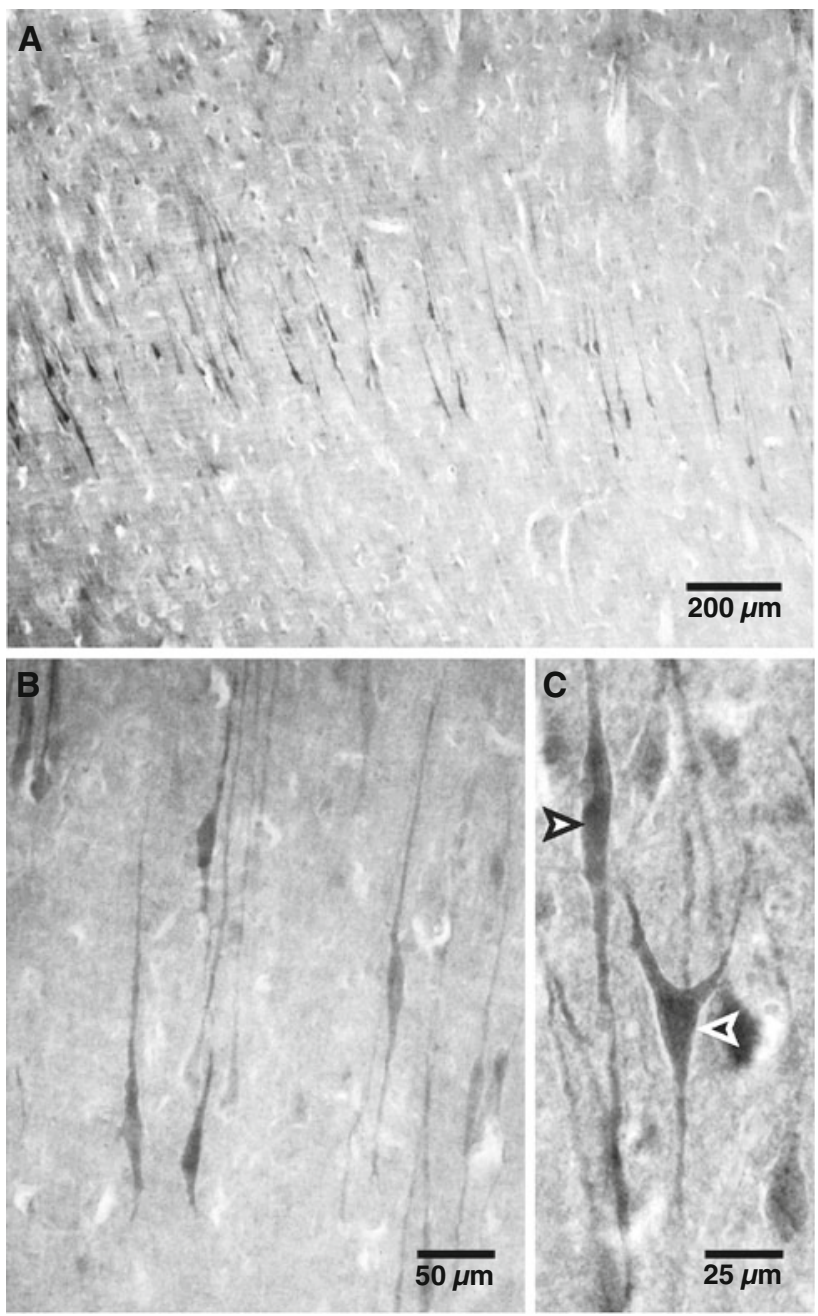

Fig. 10 a A low-power photomicrograph of immunocytochemical staining for NMB in layer 5 neurons of area FI in a 51-year-old human male subject; note the weak staining in the other cortical layers. b Most of the stained neurons are VENs in layer 5. c NMB staining of a VEN (white arrowhead) and a fork cell (black arrowhead) in layer 5 of the same subject. The cause of death in this subject was myocardial infarction. The horizontal striations are an artifact of vibratome sectioning

shape of the VENs with sometimes undulating apical and basal dendrites closely resembles that of migrating neurons with undulating leading and trailing processes, and this is particularly evident in infant brains (Allman et al. 2002). Although there are many technical difficulties in experimentally resolving whether the VENs arise by transformation or postnatal neurogenesis, future research should reveal whether either of these possibilities is correct. Hayashi et al. (2001) observed VENs in the anterior cingulate cortex of a 224 day post-conception fetal chimpanzee. This is about 2 weeks before full term (237 days) in the chimpanzee and is consistent with our observation that the VENs are present at this late stage of fetal development 


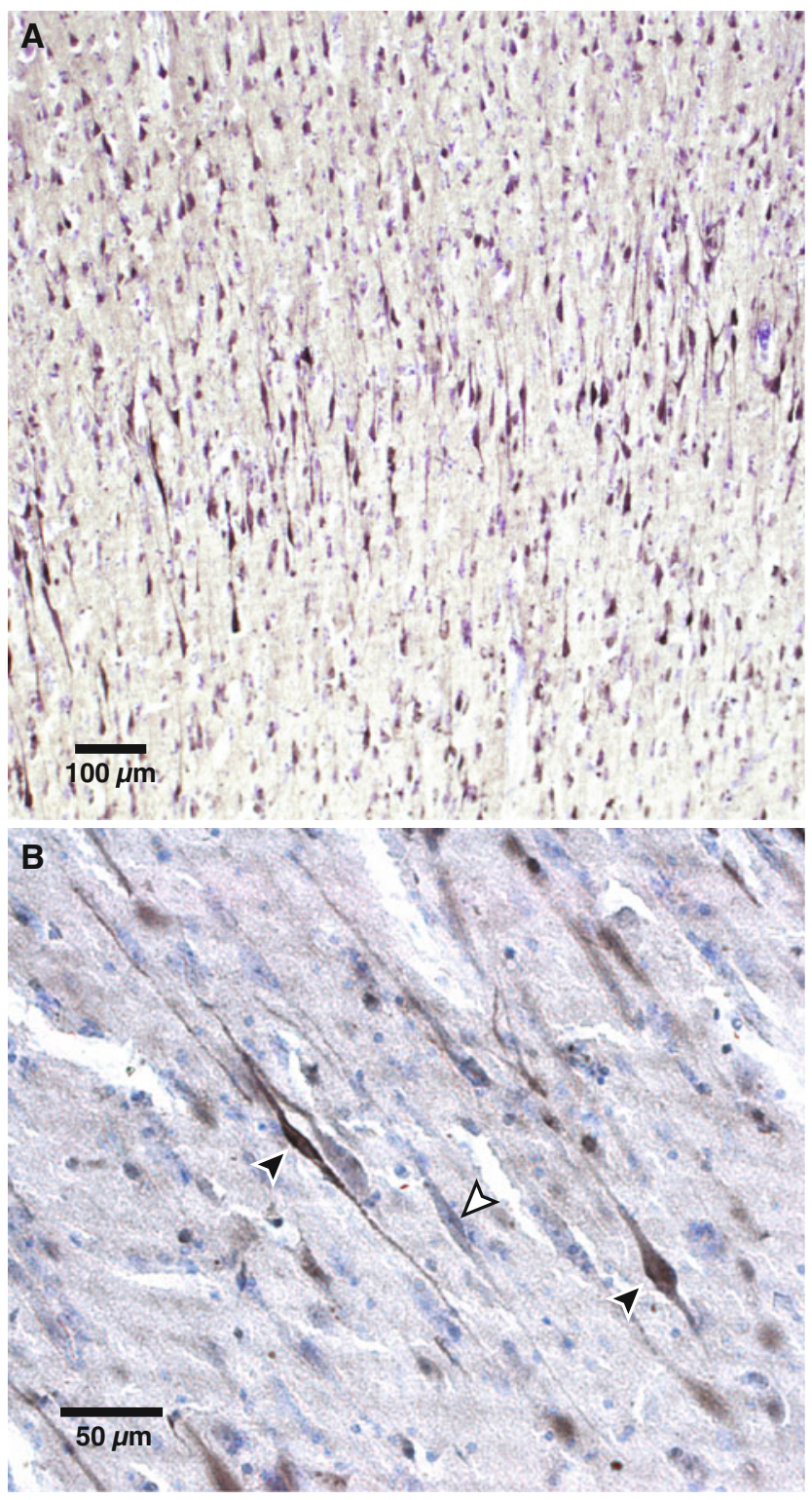

Fig. 11 a A low-power photomicrograph of immunocytochemical staining for DISC1 (brown-black) in area FI of a 51-year-old human male subject. This section has also been counterstained for cell bodies with cresyl violet; cells labeled only by the cresyl violet stain are purple-blue rather than dark brown. Note that the VENs are strongly positive for DISC1, whereas many cells in other layers are not. Some fork cells are also DISC1 positive. b A higher power image of layer 5 illustrating that the VENs and a subset of pyramidal neurons are labeled with the antibody, whereas most pyramidal neurons are unlabeled, visible only due to the cresyl violet counterstain. The black arrowheads indicate DISC1-positive VENs; the white arrowhead indicates a VEN not labeled by the antibody to DISC1

in humans. However, they also reported that $5.3 \%$ of the neurons in layer $5 \mathrm{~b}$ were VENs in this fetal chimpanzee, while in humans the VENs are rare at this stage, suggesting that the VEN population develops earlier in chimpanzees than in humans.
Hemispheric differences

An important finding in our study is the larger number of VENs in the right hemisphere than the left except in very young subjects, and thus that the rightward asymmetry emerges during the first few months of postnatal life. Stereological evidence for hemispheric differences in neuron number in primates (including humans) is very limited. Uylings et al. (2006) found a trend toward a larger number of total neurons in Broca's area for a sample of five female brains. Sherwood et al. (2007) found that right-left differences in the density of parvalbumin-positive interneurons in layers 2 and 3 of primary motor cortex in chimpanzees is linked to hand preference. However, there is excellent evidence that the anterior cingulate cortex is larger in volume on the right side from a structural MRI study of 100 young adult subjects. This study found that ACC is $13 \%$ larger on the right side, while the size of posterior cingulate cortex is the same in both hemispheres (Gündel et al. 2004). There is also structural MRI data for 142 young adults which suggest that FI is enlarged by about the same amount on the right side (Watkins et al. 2001).

The significantly increased number of VENs in the right hemisphere in FI and ACC are among the few demonstrations of hemispheric differences in neuron number based on stereological techniques, and these rightward predominances correspond to size differences in FI and ACC observed in structural MRI studies done in large populations of adult human subjects. The fact that these hemispheric differences are present both in humans and in great apes suggests that they may have existed in the common ancestor of both groups. There is recent evidence from a developmental MRI study based on 358 subjects that the anterior insula and posterior orbito-frontal cortex is thinner on the right side than the left at age 4 in normal subjects, but progresses by age 20 to be significantly thicker by about $0.3 \mathrm{~mm}$ on the right side than the left (Shaw et al. 2009). The rightward asymmetry in cortical thickness for the region containing FI in the adult brain is consistent with previous MRI studies done in adults and with the rightward asymmetry in VEN numbers in our study; however, in our study we found a rightward asymmetry in VEN numbers throughout postnatal life. Thus, the rightward predominance of VEN numbers develops before the predominance in cortical thickness in this cortical region. In our preliminary study of tractography based on diffusion tensor imaging, we found that area FI in a gorilla is connected via the uncinate fasciculus with the temporal pole, amygdala, and inferotemporal cortex. Diffusion tensor imaging provides a strong measure of the degree of coherence of axonal pathways in the brain (fractional anisotropy) and recently James Rilling et al. (personal 

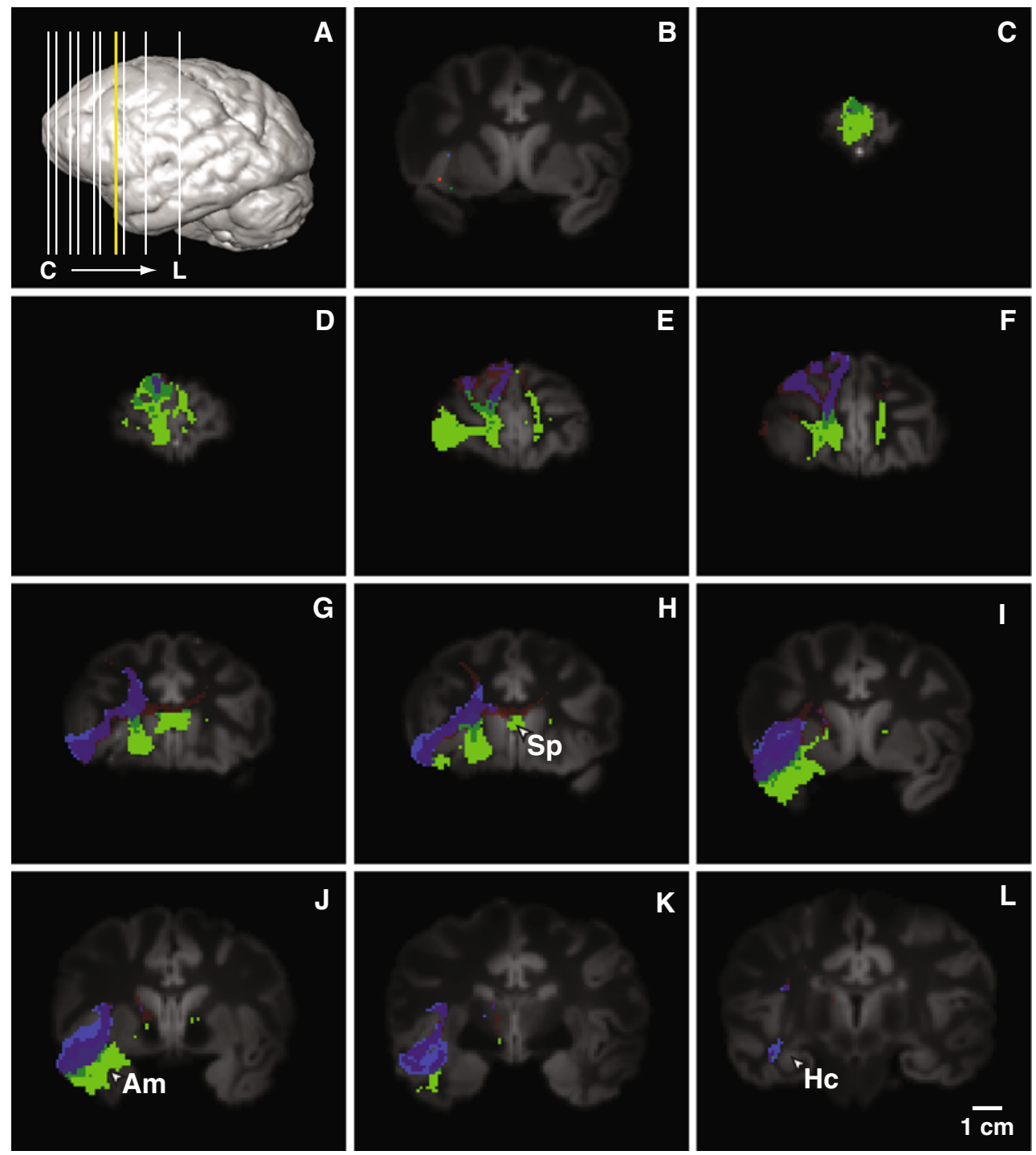

Fig. 12 Tractography based on diffusion tensor imaging of the brain of a 27-year-old male gorilla. a The levels for the sections shown in $\mathbf{b}$ through 1. The yellow line corresponds to the plane containing the seed voxels shown in $\mathbf{b}$. This plane is at the level of the section shown in i. The tractography seeds were $1 \mathrm{~mm}$ cubic voxels, placed at approximately the level of the section through FI of this gorilla shown in Fig. 3. The connections for the medial seed in FI are illustrated in green; the connections for the lateral FI seed are in red; the connections for the superior anterior insula seed are in blue. All connections are shown. In places where a voxel contains connections from multiple seeds, the colors are mixed (e. g. red plus blue yields purple). The right side of each image corresponds to the right side of the brain

result from negative feedback and subsequent error correcting behavior; the left hemisphere is preferentially involved in parasympathetic activity associated with reduced tension or calming responses (Craig 2005). Following on from this reasoning, there may be more VENs on the right side because the responses to negative feedback require more complex and more urgent behavioral responses than do situations that are calming and involve reduced tension. Many of these experiments probably have in common a right FI response, such as that which has been specifically linked to sympathetic arousal as measured by the galvanic skin response (Critchley et al. 2000). 
A meta-analysis of co-activation of amygdala and insula involving 955 responses in 86 papers reported co-activation between the amygdala and inferior anterior insula on both sides, but found it to be more pronounced on the right (Mutschler et al. 2009). In a meta-analysis of 23 functional imaging studies conducted in children and in adolescents performing various executive functioning tasks, such as go versus nogo, which typically involve intense focus and self-control, Houdé et al. (2010) found that in children the most consistent site of activation was in anterior insula on the left side, while in adolescents the most consistent site of activation was the inferior anterior insula corresponding to FI on the right side. Thus, the right FI becomes strongly engaged in executive functioning and self-control in adolescents. Houdé et al. (2010, p 6) say this change: "is consistent with the fact that adolescents are often psychologically embedded in a period of great emotional reactivity and sensitivity with negative feelings. Recognizing the necessity of being wrong is necessary to achieve high levels of adult adaptation and maturity in cognitive control. Our result might reflect a key transition around the time of adolescence toward increased influence of negative feedback (i.e. error detection and/or anticipation) on cognitive control."

There is also some evidence of preferential leftward activation in FI and ACC during positive and calming emotions (Craig 2009). The left anterior cingulate cortex was preferentially activated when subjects relaxed and reduced their sympathetic arousal through biofeedback (Critchley et al. 2001b). The right hemisphere of the brain is related to sympathetic arousal and the left hemisphere to parasympathetic quietude (Wittling 1995; Rogers and Andrew 2002; Craig 2005). This autonomic asymmetry is consistent with the proposal that the right hemisphere responds to the unexpected and the left hemisphere to more routine stimuli (MacNeilage et al. 2009). There is also evidence for this autonomic asymmetry from electrical stimulation of the insular cortex on the right and left sides in human subjects (Oppenheimer et al. 1992). Craig (2005) suggests that sympathetic activation on the right side and consequent energy expenditure by the organism, and parasympathetic activation on the left and energy conservation together function to serve as a balancing mechanism for managing the organism's energy resources. These mechanisms involve in part highly conserved circuits in the vagal complex that regulate respiration and the production of vocalizations throughout vertebrates (Bass et al. 2008).

\section{NMB expression in the VENs}

Perhaps, the most basic function of the brain is to regulate the intake of food into the gut so that nutritious substances are ingested and toxic substances are rejected or ejected.
This is probably why the brain is located near the entrance to the gut (Allman 2000). The gut has its own hormonal and neuronal mechanisms for the coordination of food processing, and the bombesin peptides NMB and GRP are crucially involved in the release of digestive enzymes, in smooth muscle contractions in peristalsis, and in the mounting of immune responses to potentially damaging ingested substances (Jensen et al. 2008). NMB and GRP participate in the local control of the gut, but these genes and their products also participate in two higher levels of gut control, first in the hypothalamus, where the digestive processes are integrated with other homeostatic systems of the body, and second in the insular and related cortices, where gut feelings and the control of the gut interact with circuitry involved in awareness, motivation, and conscious decision-making. In this context, one aspect of the functioning of the insular cortex might be as an encephalization of autonomic control. Thus, our bodies have three interacting systems for the control of the gut: one in the gut itself, one in the hypothalamus for automatic homeostatic control, and one in the cortex closely linked to selfawareness, motivation, and decision-making. The capacity for self-awareness appears to be linked to social awareness (empathy), and is reduced in autism spectrum disorders (Lombardo et al. 2007).

The expression of NMB and GRP is an evolutionarily conservative aspect of the VENs and related neurons that reflects very basic functions of the gut and appetite control. Dr. John Morris (personal communication) made us aware of the fact that NMB mRNA is expressed in a very restricted population of neurons in the deep layers of the insula in mice (see Allen Brain Atlas, NMB coronal sections at http://mouse.brain-map.org). We found that the NMB protein is expressed selectively in VENs and a subset of other layer 5 neurons in FI in humans (see Fig. 10). GRP mRNA has a similar pattern of expression in mice, and its protein is also selectively expressed on the VENs and a subset of other layer 5 neurons. The selective expression of NMB and GRP in mice and humans suggests that it may be possible to immunocytochemically identify cell populations related to VENs in mice, despite the cells not being morphologically identifiable as VENs. This raises the possibility that this VEN-related cell population in mice could be used for a wide variety of experimental investigations. The NMB-labeled population in human FI includes another morphologically distinctive neuron type, the fork cells (see Fig. 10c). The fork cells were observed as a distinct neuronal type in the human insular cortex by Ngowyang (1932). These cells closely resemble the VENs, but have an apical dendrite divided into two instead of a single apical dendrite. Thus, the VENs and fork cells may represent morphological specializations of an ancient cell type found in insular cortex in all mammals. 
The VENs are prime targets for pathology in FTD during the early stage of the illness, which is characterized by the loss of social awareness and empathy (Seeley et al. 2006, 2007; Seeley 2008). However, FTD is also frequently associated with compulsive eating (Woolley et al. 2007), and FI is significantly reduced on the right side in the FTD patients who are compulsive overeaters (Seeley 2008). These FTD findings may reflect a basic role for the VENs, fork cells, and related circuitry in the control of appetite. The anterior insula/FI is activated when subjects view facial expressions of disgust (Phillips et al. 1997; Jabbi et al. 2008). Disgust (literally, bad taste) links appetite control and social feedback, which we believe are two key functions of these circuits. The experience and expression of disgust are intense forms of negative feedback in both the consumption of food and in social interactions. Components of circuitry for the regulation of the social emotions may be a direct evolutionary outgrowth from insular circuity involved in appetite regulation and other aspects of physiological homeostasis (Woodward and Allman 2007; Williamson and Allman in press). The circuitry for the regulation of appetite through the experiences of lust and disgust may have been the evolutionary template for dichotomous social emotions, such as love and hate, gratitude and resentment, empathy and disdain, and atonement and guilt. The experiencing of these dichotomous social emotions which range from the poles of prosocial to antisocial feelings and thus regulate social distance is analogous to the regulation of food consumption through the experience of lust versus disgust. As reviewed earlier, the experience of many of these social emotions activates anterior insula. Thus, the VENs and related insular circuitry may be involved in monitoring changes in the physiological network of an individual's own body and that individual's social network. In each case, the VENs may initiate homeostatic corrections to changes in network states.

In his "somatic marker hypothesis", Damasio (1994) proposed that the monitoring of sensations arising from the gut is crucial to adaptive decision-making. The presence of $\mathrm{NMB}, \mathrm{GRP}$, and the serotonin $2 \mathrm{~b}$ receptor, which are rare in the cortex, but very common in the viscera, suggests an extension of the concept that FI and ACC are monitoring the activity in the gut (Allman et al. 2005). Possibly the expression of NMB, GRP, and the serotonin $2 b$ receptors in the VENs represents a transposition of this activity from the gut to the cortex, as components of a neural model of how the gut would react under given circumstances. Such a model would enable the organism to react more quickly to changing conditions than if it depended solely on monitoring sensations arising from the gut itself (Allman et al. 2005). Disruption of this feedback could also underlie the recently recognized role of the insula in the feelings of craving associated with addiction to food or drugs (Naqvi and Bechara 2009).
DISC1 expression in the VENs

The selective expression of the protein made by the gene DISC1 (disrupted in schizophrenia) on the VEN soma and dendrites has several interesting implications. Duan et al. (2007) blocked the expression of the DISC1 gene in postnatally generated neurons in the dentate gyrus of the mouse hippocampus through the use of selective interference with DISC1 RNA. When DISC1 expression is blocked in these neurons, there is considerably more branching of the secondary and tertiary dendrites than when DISC1 expression is normal. Thus, DISC1 reduces secondary and tertiary dendritic branching in postnatally generated neurons, and DISC1 could be part of the genetic circuitry responsible for the relative absence of higher order branching in the VENs. Crespi et al. (2007) showed that several parts of the DISC1 gene have undergone substantial positive selective changes since hominoids diverged from other anthropoids and since humans diverged from other hominoids. The site of the largest evolutionary divergence maps to exons 1 and 2 in the part of the DISC1 gene which binds to MAP1a (microtubule associated protein 1a), which is involved in the control of activity-dependent dendritic branching (Szebenyi et al. 2005). A single nucleotide polymorphism in another part of the DISC1 gene is linked to increased risks of major depression and schizophrenia, and this polymorphism is also linked to reduced volume of ACC in a large MRI study (Hashimoto et al. 2006). Variations in the DISC1 gene have been frequently associated with changes in cognitive functioning, particularly with respect to tasks that involve maintaining and shifting attention (see Chubb et al. 2008 for a review). Thus, DISC1 is a gene that has undergone considerable recent evolution and may be related to enhanced cognition in humans. DISC1 is expressed selectively on the soma and dendrites of the VENs and may be related to their distinctive dendritic architecture. The recent evolutionary changes associated with the VENs and DISC1 may carry increased vulnerabilities to dysfunction which may manifest in several neuropsychiatric disorders (Allman et al. 2005; Crespi et al. 2007; Seeley et al. 2006; 2007; Seeley 2008; Kaufman et al. 2008; van Kooten 2008; Simms et al. 2009; Williamson and Allman in press). Further investigation of gene expression in the VENs could provide insights into the dysfunctions in neuropsychiatric illnesses and also into the recent VEN evolution.

\section{DTI connections of FI and superior AI}

In our preliminary study of the connections of FI and superior AI in the brain of a gorilla, we found that the FI seeds had connections with the frontal polar cortex, while such connections were sparse from the superior AI seed. In 
humans, frontal polar cortex is involved in retrieval from episodic memory, multi-tasking, and mentalizing behaviors (Gilbert et al. 2006). Both FI and superior AI seeds were extensively connected with more posterior parts of the frontal lobe as well as much of insular cortex, and both delineated tracts just medial to the hippocampus suggesting connection with that structure. The medial FI seed is also connected with the amygdala and the septum. Septal activity is associated with the building of trust in human subjects (Krueger et al. 2007). The activity of FI is also directly associated with trust (Singer et al. 2004b). More broadly, the septum is linked to social memory and to the control of anterior hypothalamic functions mediated by vasopressin and oxytocin involved in parental care and social attachment (Numan 2000; Lim and Young 2006). The VENs in FI are likely to project to some or all of the structures revealed by the tractography.

Acknowledgments The authors would like to thank Dr. Barbara Wold, Dr. Chet Sherwood, Dr. Bill Seeley, Dr. James Rilling, and Dr. A. D. Craig for their invaluable comments and discussion. We thank Dr. Micheal Tyszka and Dr. Jason Kaufman for the MRI imaging of the ape brains. We thank Dr. Kristen Tillisch and Dr. Emeran Mayer for the MR images of the young adult human subject. We are grateful to Dr. John Morris for his suggestion that the expression of neuromedin B and gastrin releasing peptide in the mouse insular cortex might be related to the VENs. We thank Dr. Heidi Griffith for her help in collecting some of the human stereological data. We thank Archibald Fobbs, curator of the Yakovlev and Welker Brain Collections and Dr. Adrianne Noe, Director, National Museum of Health and Medicine for their crucial role in preserving these collections and making them available to us and to the broader scientific community. In the Hof lab, technical help was provided by B. Wicinski and S. Harry. Several of the great ape brains involved in this study were on loan to the "Great Ape Aging Project" from zoological gardens that are accredited by the Association of Zoos and Aquariums (AZA) and that participate in the Ape Taxon Advisory Group (Ape-TAG). We especially appreciate the contribution of zoo veterinarians and staff in collecting and providing specimens. Additional human tissue was obtained from the NICHD Brain and Tissue Bank for Developmental Disorders. Some comparative specimens were collected under the "Comparative Neurobiology of Aging Resource," NIH/NIA grant AG14308, J. Erwin, PI. This research was supported by the James S. McDonnell Foundation, the David and Lucille Packard Foundation, and the Simons Foundation.

Open Access This article is distributed under the terms of the Creative Commons Attribution Noncommercial License which permits any noncommercial use, distribution, and reproduction in any medium, provided the original author(s) and source are credited.

\section{References}

Allman JM (2000) Evolving brains. Scientific American LibraryW.H.Freeman, New York

Allman J, McLaughlin T, Hakeem A (1993) Brain weight and lifespan in primate species. Proc Natl Acad Sci USA 90:118-122

Allman JM, Hakeem A, Erwin JM, Nimchinsky E, Hof P (2001) Anterior cingulate cortex: the evolution of an interface between emotion and cognition. Ann NY Acad Sci 935:107-117
Allman J, Hakeem A, Watson K (2002) Two phylogenetic specializations in the human brain. Neuroscientist 8:335-346

Allman J, Watson K, Tetreault N, Hakeem A (2005) Intuition and autism: a possible role for von Economo neurons. Trends Cogn Sci 9:367-373

Aziz-Zadeh L, Kaplan J, Iocoboni M (2009) "Aha": the neural correlates of verbal insight solutions. Hum Brain Mapp 30:908916

Barger N, Stefanacci L, Semendeferi K (2007) A comparative volumetric analysis of the amygdaloid complex and basolateral division in the human and ape brain. Am J Phys Anthropol 134:392-403

Baron-Cohen S, Ring HA, Wheelwright S, Bullmore ET, Brammer MJ, Simmons A, Williams SC (1999) Social intelligence in the normal and autistic brain: an fMRI study. Eur J Neurosci 11:1891-1898

Bartels A, Zeki S (2004) The neural correlates of maternal and romantic love. Neuroimage 21:1155-1166

Bass AH, Gilland EH, Baker R (2008) Evolutionary origins for social vocalization in a vertebrate hindbrain-spinal compartment. Science 321:417-421

Berthoz S, Armony JL, Blair RJR, Dolan RJ (2002) An fMRI study of intentional and unintentional (embarrassing) violations of social norms. Brain 125:1696-1708

Betz W (1881) Ueber die feinere Structur der Gehirnrinde des Menschen. Zentralbl Med Wiss 19:193-195, 209-213, 231-234

Brauer K, Schober W (1970) Catalogue of mammalian brains. VEB Gustav Fischer Verlag, Jena

Butti C, Sherwood CC, Hakeem AY, Allman JM, Hof PR (2009) Total number and volume of Von Economo neurons in the cerebral cortex of cetaceans. J Comp Neurol 515:243-259

Chubb JE, Bradshaw NJ, Soares DC, Porteous DJ, Millar JK (2008) The DISC locus in psychiatric illness. Mol Psychiatry 13:36-64

Craig AD (2003) A new view of pain as a homeostasis emotion. Trends Neurosci 26:303-307

Craig AD (2005) Forebrain emotional asymmetry: a neuroanatomical basis? Trends Cogn Sci 912:566-571

Craig AD (2009) How do you feel-now? The anterior insula and human awareness. Nat Rev Neurosci 10:59-70

Craig AD (2010) The sentient self. Brain Struct Funct. doi: 10.1007/s00429-010-0248-y

Crespi B, Summers K, Dorus S (2007) Adaptive evolution of genes underlying schizophrenia. Proc Roy Soc B 274:2801-2810

Critchley HD, Elliott R, Mathias CJ, Dolan RJ (2000) Neural activity relating to generation and representation of galvanic skin conductance responses. J Neurosci 20:3033-3040

Critchley HD, Mathias C, Dolan R (2001a) Neural activity in the human brain relating to uncertainty and arousal during anticipation. Neuron 29:537-545

Critchley HD, Melmed RN, Featherstone E, Mathias CJ, Dolan RJ (2001b) Brain activity during biofeedback relaxation: a functional neuroimaging investigation. Brain 124:1003-1012

Croxson PL, Johansen-Berg H, Behrens TE, Robson MD, Pinsk MA, Gross CG, Richter W, Richter MC, Kastner S, Rushworth MF (2005) Quantitative investigation of connections of the prefrontal cortex in the human and macaque using probabilistic diffusion tractography. J Neurosci 28:8854-8866

Damasio A (1994) Descartes error. Putnam, New York

Dehaene S, Posner MI, Tucker DM (1994) Localization of a neural system for error detection and compensation. Psychol Sci 5:303-305

Di Martino A, Ross K, Uddin L, Sklar A, Castellanos F, Milham M (2009) Processes in autism spectrum disorders: an activation likelihood estimation meta-analysis. Biol Psychiatry 65:63-74

Dosenbach NU, Fair DA, Miezin FM, Cohen AL, Wenger KK, Dosenbach RA, Fox MD, Snyder AZ, Vincent JL, Raichle ME, 
Schlaggar BL, Petersen SE (2007) Distinct brain networks for adaptive and stable task control in humans. Proc Natl Acad Sci USA 104:11073-11078

Duan X, Chang JH, Ge S, Faulkner RL, Kim JY, Kitabatake Y, Liu $\mathrm{XB}$, Yang CH, Jordan JD, Ma DK, Liu CY, Ganesan S, Cheng HJ, Ming GL, Lu B, Song H (2007) Disrupted-in-schizophrenia 1 regulates integration of newly generated neurons in the adult brain. Cell 130:1146-1158

Galdikas BMF (1985) Orangutan sociality at Tanjung Puting. Am J Primatol 9:101-119

Gallyas F (1971) A principle for silver staining of tissue elements by physical development. Acta Morphol Acad Sci 19:57-71

Gehring WJ, Goss B, Coles MGH, Meyer DE, Donchin E (1993) A neural system of error detection and compensation. Psychol Sci 4:385-390

Gilbert S, Spengler S, Simons J, Steele J, Lawrie S, Frith C, Burgess P (2006) Functional specialization within rostral prefrontal cortex (Area 10): a meta-analysis. J Cogn Neurosci 18:932-948

Gilles FH, Leviton A, Dooling EC (1983) Developing human brain, growth and epidemiologic neuropathology. John Wright - PSG Inc, Boston

Gündel H, López-Sala A, Ceballos-Baumann AO, Deus J, Cardoner N, Marten-Mittag B, Soriano-Mas C, Pujol J (2004) Alexithymia correlates with the size of right anterior cingulate. Psychosom Med 66:132-140

Hakeem AY, Sherwood CC, Bonar CJ, Butti C, Hof PR, Allman JM (2009) Von Economo neurons in the elephant brain. Anat Rec (Hoboken) 292:242-248

Hammarberg C (1895) Studien über Klinik und Pathologie der Idiotie nebst Untersuchungen über die normale Anatomie des Hirnrinde. Berling, Uppsala

Harrison NA, Gray MA, Critchley HD (2009) Dynamic pupillary exchange engages brain regions encoding social salience. Soc Neurosci 4:233-243

Hashimoto R, Numakawa T, Ohnishi T, Kumamaru E, Yagasaki Y, Ishimoto T, Mori T, Nemoto K, Adachi N, Izumi A, Chiba S, Noguchi H, Suzuki T, Iwata N, Ozaki N, Taguchi T, Kamiya A, Kosuga A, Tatsumi M, Kamijima K, Weinberger DR, Sawa A, Kunugi H (2006) Impact of the DISC1 Ser704Cys polymorphism on risk for major depression, brain morphology and ERK signaling. Hum Mol Genet 15:3024-3033

Hayashi M, Ito M, Shimizu K (2001) The spindle neurons are present in the cingulate cortex of chimpanzee fetus. Neurosci Lett 309:97-100

Hof PR, Van der Gucht E (2007) Structure of the cerebral cortex of the humpback whale, Megaptera novaeangliae (Cetacea, Mysticeti, Balaenopteridae). Anat Rec 290:1-31

Houdé O, Rossi S, Lubin A, Joliot M (2010) Mapping numerical processing, reading, and executive functions in the developing brain: an fMRI meta-analysis of 52 studies including 842 children. Dev Sci 1:1-10

Jabbi M, Bastiaansen J, Keysers C (2008) A common anterior insula representation of disgust observation, experience and imagination shows divergent functional connectivity pathways. PLos One 8:e2939

Jensen RT, Battey JF, Spindel ER, BEnya RV (2008) International Union of Pharmacology, LXVIII. Mammalian bombesin receptors: nomenclature, distribution, pharmacology, signaling, and functions in normal and disease states. Pharmacol Rev 60:1-42

Kaufman JA, Paul LK, Manaye KF, Granstedt AE, Hof PR, Hakeem AY, Allman JM (2008) Selective reduction of Von Economo neuron number in agenesis of the corpus callosum. Acta Neuropathol 116:479-489

Kennedy DP, Semendeferi K, Courchesne E (2007) No reduction of spindle neuron number in frontoinsular cortex in autism. Brain Cogn 64:124-129
Kikyo H, Ohki K (2002) Neural correlates for feeling-of-knowing: an fMRI parametric analysis. Neuron 36:177-186

Klein TA, Endrass T, Kathmann N, Neumann J, von Cramon DY, Ullsperger M (2007) Neural correlates of error awareness. NeuroImage 34:1774-1781

Krueger F, McCabe K, Moll J, Kriegeskorte N, Zahn R, Strenziok M, Heinecke A, Grafman J (2007) Neural correlates of trust. Proc Natl Acad Sci USA 104:20084-20089

Kunimatsu Y, Nakatsukasa M, Sawada Y, Sakai T, Hyodo M, Hyodo H, Itaya T, Nakaya H, Saegusa H, Mazurier A, Saneyoshi M, Tsujikawa H, Yamamoto A, Mbua E (2007) A new Late Miocene great ape from Kenya and its implications for the origins of African great apes and humans. Proc Natl Acad Sci USA 104:19220-19225

Lamm C, Singer T (2010) Role of anterior insular cortex in social emotions. Brain Struct Funct. doi:10.1007/s00429-010-0251-3

Lim M, Young L (2006) Neuropeptidergic regulation of affiliative behavior and social bonding in animals. Horm Behav 50:506517

Lombardo MV, Barnes JL, Wheelwright SJ, Baron-Cohen S (2007) Self-referential cognition and empathy in autism. PLoS One 2:e883

Lorberbaum JP, Newman JD, Horwitz AR, Dubno JR, Lydiard RB, Hamner MB, Bohning DE, George MS (2002) A potential role for thalamocingulate circuitry in human maternal behavior. Biol Psychiatry 51:431-445

MacNeilage PF, Rogers LJ, Vallortigara G (2009) Origins of the left \& right brain. Sci Am 301:60-67

Merker B (1983) Silver staining of cell bodies by means of physical development. J Neurosci Methods 9:235-241

Mitani JC, Grether GF, Rodman PS, Priatna D (1991) Associations among wild orangutans: sociality, passive aggregations or chance? Anim Behav 42:33-46

Mutschler I, Wieckhorst B, Kowalevski S, Derix J, Wentlandt J, Schulze-Bonhage A, Ball T (2009) Functional organization of the human interior insular cortex. Neurosci Lett 457:66-70

Naqvi NH, Bechara A (2009) The hidden island of addiction: the insula. Trends Neurosci 32:56-67

Nelson S, Dosenbach, N, Cohen, A, Schlaggar B, Petersen S (2010) Role of the anterior insula in task-level control and focal attention. Brain Struct Funct. doi:10.1007/s00429-010$0260-2$

Ngowyang G (1932) Beschreibung einer Art von Spezialzellen in der Inselrinde - zugleich Bemerkungen über die v. Economoschen Spezialzellen. J Psychol Neurol 44:671-674

Nimchinsky EA, Vogt BA, Morrison JH, Hof PR (1995) Spindle neurons of the human anterior cingulate cortex. J Comp Neurol 355:27-37

Nimchinsky E, Gilissen E, Allman J, Perl D, Erwin J, Hof P (1999) A neuronal morphologic type unique to humans and great apes. Proc Nat Acad Sci USA 96:5268-5273

Numan R (2000) The behavioral neuroscience of the septal region. Springer, New York

Oppenheimer SM, Gelb A, Girvin JP, Hachinski VC (1992) Cardiovascular effects of human insular cortex stimulation. Neurology 42:1727-1732

Patterson F, Gordon W (2002) Twenty-seven years of Project Koko and Michael. In: Galdikas B, Briggs N, Sheeran L, Shapiro G, Goodall J (eds) All apes great and small, vol I. Kluwer Press, New York, pp 165-176

Paul LK, Brown WS, Adolphs R, Tyszka JM, Richards LJ, Mukherjee P, Sherr EH (2007) Agenesis of the corpus callosum: genetic, developmental and functional aspects of connectivity. Nat Rev Neurosci 8:287-299

Phillips ML, Young AW, Senior C, Brammer M, Andrew C, Calder AJ, Bullmore ET, Perrett DI, Rowland D, Williams SC, Gray JA, 
David AS (1997) A specific neural substrate for perceiving facial expressions of disgust. Nature 389:495-498

Ploran E, Nelson S, Velanova K, Petersem S, Wheeler M (2007) Evidence accumulation and moment of recognition: dissociating perceptual recognition processes using fMRI. J Neurosci 27:11012-11924

Preuschhoff K, Quartz S, Bossaerts P (2008) Human insula activation reflects risk prediction errors as well as risk. J Neurosci 28:27452752

Ramón y Cajal S (1899) Textura del Sistema Nervioso del Hombre y de los Vertebrados, Tomo II. Nicolás Moya, Madrid

Rankin KP, Gorno-Tempini ML, Allison SC, Stanley CM, Glenn S, Weiner MW, Miller BL (2006) Structural anatomy of empathy in neurodegenerative disease. Brain 129:2945-2956

Rilling J, Goldsmith D, Glenn A, Jairam M, Elfenbein H, Dagenais J, Murdock C, Pagnoni (2008) Neural correlates of the affective response to unreciprocated cooperation. Neuropsychologia 46:1265-1266

Rogers L, Andrew R (2002) Comparative vertebrate lateralization. Cambridge University Press, Cambridge

Rose M (1927) Gyrus limbicus anterior und Regio retrosplenialis (Cortex holoprotoptychos quinquestratificatus) - Vergleichende Architektonik bei Tier und Menschen. J Psychol Neurol 35:5-217

Rose M (1928) Die Inselrinde des Menschen und der Tiere. J Psychol Neurol 37:467-624

Sanfey AG, Rilling RJ, Aronson JA, Nystrom LE, Cohen JD (2003) The neural basis of economic decision-making in the ultimatum game. Science 300:1755-1758

Schenker NM, Desgouttes A, Semendeferi K (2005) Neural connectivity and cortical substrates of cognition in hominoids. J Hum Evol 49:547-569

Seeley WW (2008) Selective functional, regional, and neuronal vulnerability in frontotemporal dementia. Curr Opin Neurol 21:701-707

Seeley WW, Carlin DA, Allman JM, Macedo MN, Bush C, Miller BL, Dearmond SJ (2006) Early frontotemporal dementia targets neurons unique to apes and humans. Ann Neurol 60:660-667

Seeley WW, Allman JM, Carlin DA, Crawford RK, Macedo MN, Greicius MD, Dearmond SJ, Miller BL (2007) Divergent social functioning in behavioral variant frontotemporal dementia and Alzheimer disease: reciprocal networks and neuronal evolution. Alzheimer Dis Assoc Disord 21:S50-S57

Semendeferi K, Armstrong E, Schleicher A, Zilles K, Van Hoesen GW (1998) Limbic frontal cortex in hominoids: a comparative study of area 13. Am J Phys Anthropol 106:129-155

Shaw P, Lalonde F, Lepage C, Rabin C, Eckstrand K, Sharp W, Greenstein D, Evans A, Giedd JM, Rapoport J (2009) Development of cortical asymmetry in typically developing children and its disruption in attention-deficit/hyperactivity disorder. Arch Gen Psychiatry 66:888-896

Sherwood CC, Holloway RL, Erwin JM, Hof PR (2004) Cortical orofacial motor representation in Old World monkeys, great apes, and humans. II: stereologic analysis of chemoarchitecture. Brain Behav Evol 63:82-106

Sherwood CC, Wahl E, Erwin JM, Hof PR, Hopkins WD (2007) Histological asymmetries of primary motor cortex predict handedness in chimpanzees. J Comp Neurol 503:525-537

Shin LM, Dougherty DD, Orr SP, Pitman RK, Lasko M, Macklin ML, Alpert NM, Fischman AJ, Rauch SL (2000) Activation of anterior paralimbic structures during guilt-related script-driven imagery. Biol Psychiatry 48:43-50

Shorvon S (2005) Handbook of epilepsy treatment. Wiley, New York

Simms ML, Kemper TL, Timbie CM, Bauman ML, Blatt GJ (2009) The anterior cingulate cortex in autism: heterogeneity of qualitative and quantitative cytoarchitectonic features suggests possible subgroups. Acta Neuropathol 118:673-684

Singer T, Seymour B, O’Doherty J, Kaube H, Dolan RJ, Frith CD (2004a) Empathy for pain involves the affective but not sensory components of pain. Science 303:1157-1162

Singer T, Kiebel SJ, Winston JS, Dolan RJ, Frith CD (2004b) Brain responses to the acquired moral status of faces. Neuron 41:653662

Singleton I, van Schaik CP (2002) The social organisation of a population of Sumatran orangutans. Folia Primatol 73:1-20

Spence SA, Farrow TF, Herford AE, Wilkinson ID, Zheng Y, Woodruff PW (2001) Behavioural and functional anatomical correlates of deception in humans. NeuroReport 12:2849-2853

Sridharan D, Levitin DJ, Menon V (2008) A critical role for the right fronto-insular cortex in switching between central-executive and default-mode networks. Proc Natl Acad Sci USA 105:12569_ 12574

Stephan H, Frahm HD, Baron G (1981) New and revised data on volumes of brain structures in insectivores and primates. Folia Primatol 35:1-29

Szebenyi G, Bollati F, Bisbal M, Sheridan S, Faas L, Wray R, Haferkamp S, Nguyen S, Caceres A, Brady S (2005) Activitydriven dendritic remodeling requires microtubule-associated Protein 1a. Curr Biol 15:1820-1826

Ullsperger M, von Cramon DY (2004) Neuroimaging of performance monitoring: error detection and beyond. Cortex 40:593-604

Uylings H, Jacosen A, Zilles K, Amunts K (2006) Left-right asymmetry in volume and number of neurons in adult Broca's area. Cortex 42:652-658

van Kooten (2008) Autism counts. Dissertation, Maastricht University

von Economo C (1926) Eine neue Art Spezialzellen des Lobus cinguli und Lobus insulae. Zschr ges Neurol Psychiat 100:706-712

Von Economo C (2009) Cellular structure of the human cerebral cortex. Translated by L.C. Triarhou, Karger, Basel

Von Economo C, Koskinas G (1925) Die Cytoarchitectonik der Hirnrinde des erwachsenen Menschen. Springer, Berlin

Wang SS, Shultz JR, Burish MJ, Harrison KH, Hof PR, Towns LC, Wagers MW, Wyatt KD (2008) Functional trade-offs in white matter axonal scaling. J Neurosci 28:4047-4056

Watkins KE, Paus T, Lerch JP, Zijdenbos A, Collins DL, Neelin P, Taylor J, Worsley KJ, Evans AC (2001) Structural asymmetries in human brain: a voxel-based statistical analysis of 142 brains. Cereb Cortex 11:868-877

Watson KK, Jones TK, Allman JM (2006) Dendritic architecture of the von Economo neurons. Neuroscience 141:1107-1112

Watson KK, Matthews BJ, Allman JM (2007) Brain activation during sight gags and language-dependent humor. Cereb Cortex 17:314-324

Welker WI, Seidenstein S (1959) Somatic sensory representation in the cerebral cortex of the raccoon (Procyon lotor). J Comp Neurol 111:469-501

Williamson P, Allman JM (2010) The human illnesses: neuropsychiatric disorders and the nature of the human brain. Oxford University Press (in press)

Wittling W (1995) Brain Asymmetry in the control of autonomicphysiological activity. In: Davidson RJ, Hugdahl K (eds) Brain asymmetry. MIT Press, Cambridge, pp 305-356

Woodward J, Allman J (2007) Moral intuition: its neural substrates and normative significance. J Physiol Paris 101(4-6):179-202

Woolley JD, Gorno-Tempini ML, Seeley WW, Rankin K, Lee SS, Matthews BR, Miller BL (2007) Binge eating is associated with right orbitofrontal-insular-striatal atrophy in frontotemporal dementia. Neurology 69:1424-1433 